\title{
Tunable Cross-Linking and Adhesion of Gelatin Hydrogels via Bioorthogonal Click Chemistry
}

\author{
Nicola Contessi Negrini, Ana Angelova Volponi, Paul T. Sharpe, and Adam D. Celiz* \\ Cite This: ACS Biomater. Sci. Eng. 2021, 7, 4330-4346 \\ Read Online
}

ABSTRACT: Engineering cytocompatible hydrogels with tunable physico-mechanical properties as a biomimetic three-dimensional extracellular matrix (ECM) is fundamental to guide cell response and target tissue regeneration or development of in vitro models. Gelatin represents an optimal choice given its ECM biomimetic properties; however, gelatin cross-linking is required to ensure structural stability at physiological temperature (i.e., $T>$ $\left.T_{\text {sol-gel gelatin }}\right)$. Here, we use a previously developed cross-linking reaction between tetrazine $(\mathrm{Tz})$ - and norbornene $(\mathrm{Nb})$ modified gelatin derivatives to prepare gelatin hydrogels and we demonstrate the possible tuning of their properties by varying their degree of modification (DOM) and the $\mathrm{Tz} / \mathrm{Nb}$ ratio $(R)$. The percentage
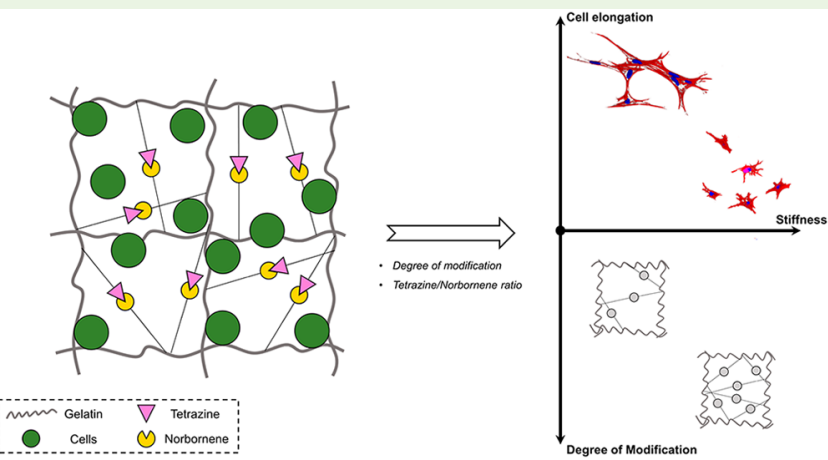
DOM of the gelatin derivatives was tuned between 5 and $15 \%$.

Hydrogels prepared with higher DOM cross-linked faster (i.e., 10-20 min) compared to hydrogels prepared with lower DOM (i.e., 60-70 $\mathrm{min}$ ). A higher $\mathrm{DOM}$ and equimolar $\mathrm{Tz} / \mathrm{Nb}$ ratio $R$ resulted in hydrogels with lower weight variation after immersion in PBS at $37^{\circ} \mathrm{C}$. The mechanical properties of the hydrogels were tuned by varying DOM and $R$ by 1 order of magnitude, achieving elastic modulus $E$ values ranging from 0.5 (low $\mathrm{DOM}$ and nonequimolar $\mathrm{Tz} / \mathrm{Nb}$ ratio) to $5 \mathrm{kPa}$ (high $\mathrm{DOM}$ and equimolar Tz/Nb ratio). Human dental pulp stem cells were embedded in the hydrogels and successfully $3 \mathrm{D}$ cultured in the hydrogels (percentage viable cells $>85 \%)$. An increase in metabolic activity and a more elongated cell morphology was detected for cells cultured in hydrogels with lower mechanical properties $(E<1 \mathrm{kPa})$. Hydrogels prepared with an excess of $\mathrm{Tz}$ or $\mathrm{Nb}$ were successfully adhered and remained in contact during in vitro cultures, highlighting the potential use of these hydrogels as compartmentalized coculture systems. The successful tuning of the gelatin hydrogel properties here developed by controlling their bioorthogonal cross-linking is promising for tissue engineering and in vitro modeling applications.

KEYWORDS: gelatin hydrogel, bioorthogonal cross-linking, degree of modification, hydrogel adhesion, dental pulp stem cell, 3D cell culture

\section{INTRODUCTION}

Hydrogels, water-swollen polymeric networks, have emerged as unique, powerful platforms for the three-dimensional (3D) culture of cells for tissue engineering, regenerative medicine, and in vitro modeling applications. ${ }^{1,2}$ Compared to traditional two-dimensional (2D) culture systems which are not representative of the in vivo cell environment, hydrogels can be designed to recreate a biomimetic $3 \mathrm{D}$ extracellular matrix (ECM) by tuning their physical, (bio)chemical, and mechanical properties. ${ }^{3}$ The selection of the polymer(s) and cross-linking method(s) used to prepare hydrogels is critical to finely tune their properties and guide a desired cellular response to achieve a $3 \mathrm{D}$ biomimetic construct.

Hydrogels can be prepared using polymers from synthetic or natural origin. Despite synthetic polymers being characterized by high reproducibility and ease in tailoring their physicomechanical properties, synthetic polymer-based hydrogels lack ECM-biomimetic motifs and the capability of being remodeled by cells. Thus, functionalization by addition of motifs to promote cell adhesion (e.g., Arg-Gly-Asp, RGD, and Tyr-IleGly-Ser-Arg, YIGSR) and cleavable groups to promote hydrogel degradation and remodeling (e.g., matrix metalloproteinases, MMP, sensitive sequences) is required to prepare bioactive synthetic polymer hydrogels, as described for poly(ethylene glycol) (PEG), ${ }^{4,5}$ poly(hydroxyethyl methacrylate) (pHEMA), ${ }^{6}$ and poly(vinyl alcohol) (PVA) hydrogels. ${ }^{7}$ Naturally derived polymer hydrogels provide a useful alternative as their structure is more reminiscent of ECM. However, natural-derived polymers are characterized by batchto-batch variability, ${ }^{3}$ potentially uncontrolled degradation, ${ }^{8}$ and weaker mechanical properties, ${ }^{9}$ making the control of the

Special Issue: Advanced Biomedical Hydrogels

Received: January 27, 2021

Accepted: May 3, 2021

Published: June 4, 2021 
hydrogel properties challenging. Moreover, decoupling the mechanical properties of the hydrogel and biological response still represents a challenge, thus limiting the potential applications of these materials. ${ }^{10,11}$ Naturally derived polymer hydrogels include polysaccharide-based (e.g., alginate, chitosan, hyaluronic acid) and protein-based hydrogels (silk, collagen, gelatin). ${ }^{12}$ Among these, gelatin has gained tremendous interest for the fabrication of tissue engineering constructs and in vitro models thanks to its unique advantages.

Gelatin is a protein obtained by partial hydrolysis of collagen. Gelatin is readily available with relatively low costs, highly versatile, it can be processed and fabricated in different forms, it maintains key collagen sequences fundamental for biomaterial-cell interactions (i.e., cell-adhesion motifs and MMP-sensitive sequences), it is water-soluble and less immunogenic compared to collagen. ${ }^{13,14}$ Gelatin dissolves in water at $T>T_{\text {sol-gel }}$ (i.e., $T_{\text {sol-gel }}$ typically around $20-30{ }^{\circ} \mathrm{C}$ ) and spontaneously forms reversible gels at $T<T_{\text {sol-gel }}$. Thus, cross-linking strategies are required to obtain mechanically and thermally stable hydrogels at physiological temperature (i.e., $T$ $\left.>T_{\text {sol-gel }}\right)$. Gelatin cross-linking can be achieved by physical, enzymatic, and chemical methods. ${ }^{15}$ Physical methods include electron beam irradiation, ${ }^{16} \gamma$-irradiation, ${ }^{17}$ and dehydrothermal treatment; ${ }^{18}$ however, using these methods generally leads to low degrees of cross-linking and mechanically weak hydrogels. Alternatively, enzymatic methods can be used by adding enzymes (e.g., microbial transglutaminase) that covalently cross-link glutamine and lysine residues in gelatin. ${ }^{19,20}$ Finally, chemical methods can be used to improve the degree of control over physico-mechanical properties of the hydrogels. Covalent bonds between the gelatin polymer chains can be formed by adding cross-linking molecules eventually removed from the hydrogel network (i.e., zero length crosslinking methods, such as carbodiimide) $)^{21}$ or by addition of cross-linking molecules incorporated into the hydrogel network (i.e., nonzero length cross-linking methods, such as glutaraldehyde, ${ }^{22}$ acrylamides, ${ }^{23,24}$ and isocyanates). ${ }^{25}$ Despite these methods allowing for improved control over hydrogels properties and for the formation of stable networks, concerns of cytotoxicity of residual cross-linking molecules, release of cytotoxic products during hydrogel degradation, and potential cross-reaction with biological proteins have been raised. ${ }^{26}$ Photo-cross-linkable gelatin derivatives (e.g., methacryloyl gelatin, GelMA $)^{27,28}$ have also been developed to allow cell encapsulation during hydrogel cross-linking, typically by addition of a photoinitiator and subsequent UV light irradiation. ${ }^{29}$ However, potential cytotoxicity of photoinitiator, inhomogeneous light depth penetration and application of UV light might affect homogeneity of the hydrogel properties and cell viability and has led to visible light-based cross-linking strategies being investigated. ${ }^{30,31}$

Recently, bioorthogonal cross-linking strategies have been developed for hydrogel cross-linking. Bioorthogonal crosslinking, by definition, is highly selective and does not interfere or react with biological systems; it occurs in physiological, mild conditions and with nontoxic effects on cells and tissues. ${ }^{32}$ As such, these strategies are particularly promising for the development of cell-laden 3D hydrogels. ${ }^{33}$ Bioorthogonal cross-linking strategies include azide-alkyne cycloaddition, nitrile oxide cycloaddition, Diels-Alder reactions, and inverseelectron demand Diels-Alder reactions. ${ }^{34}$ The latter has been investigated by using, for instance, the reaction between tetrazine $(\mathrm{Tz})$ and norbornene $(\mathrm{Nb})$, which has been demonstrated as being successful for the preparation of hydrogels based on PEG, ${ }^{35}$ alginate, ${ }^{36}$ hyaluronan, ${ }^{37}$ methylcellulose, $^{38}$ and different polymer combinations. ${ }^{39-43}$ In recent work, the suitability of this bioorthogonal cross-linking strategy was investigated for the first time for cell-laden gelatin hydrogels. $^{44}$ The authors prepared two different gelatin derivatives, functionalized either with $\mathrm{Tz}$ or $\mathrm{Nb}$, with a similar degree of modification (i.e., approximately 20\%), and mixed them to form gelatin hydrogels at different gelatin concentrations (i.e., 5 and 10\%). After studying the rheological properties of the hydrogels, the authors selected an optimal $\mathrm{Tz} / \mathrm{Nb}$ ratio to prepare the hydrogels and investigated the influence of the gelatin polymer concentration on the in vitro biological properties of the hydrogels. Promising results were achieved for the use of the prepared hydrogels as injectable, cytocompatible biomaterials. However, control over the physico-mechanical and biological properties of such hydrogels by only varying the hydrogel concentration might represent a limitation for the achievable properties, guidance of desired cell responses, and versatility of their applications. Therefore, in this work, we further study the application of the $\mathrm{Tz}-\mathrm{Nb}$ bioorthogonal cross-linking for gelatin hydrogels. We investigate the possibility of finely tuning the physico-mechanical hydrogel properties by fixing the polymer concentration and varying (1) the degree of modification of the gelatin derivatives and (2) the $\mathrm{Tz} / \mathrm{Nb}$ ratio used to prepare the hydrogel. We demonstrate that rheological properties, swelling, and mechanical properties of the hydrogels can be modulated by varying these hydrogel preparation parameters, and we demonstrate how cell response varies during $3 \mathrm{D}$ in vitro culture in different cross-linked gelatin hydrogel formulations. Furthermore, we utilize biorthogonal chemistry to adhere hydrogels to form compartmentalized structures which could be used for compartmentalized cell cocultures.

\section{EXPERIMENTAL SECTION}

Materials. Materials were purchased from Merck unless differently specified. The materials used were gelatin (X-Pure low-endotoxin type B from bovine bones, gel strength 240-270 g, Rousselot Biomedical); methyltetrazine-amine (tetrazine, Tz, BroadPharm), 5-norbornene-2methylamine (norbornene, Nb, TCI Tokyo Chemical Industry Co., Ltd.), 2-( $N$-morpholino)ethanesulfonic acid (MES), deuterium oxide containing 0.05 wt \% 3-(trimethylsilyl)propionic-2,2,3,3-d4 acid (TMSP), N-hydroxysuccinimide (NHS), N-(3-(dimethylamino)propyl)- $N^{\prime}$-ethylcarbodiimide hydrochloride (EDC, Apollo Scientific), sodium azide, Dulbecco phosphate buffered saline (PBS), polydimethylsiloxane (PDMS, SYLGARD 184), Hanks' Balanced Salt Solution (HBSS), Trypsin-EDTA solution, AlamarBlue (Thermo Fisher Scientific), Calcein-AM, propidium iodide, rhodamine phalloidin (Thermo Fisher Scientific), Hoechst 33342 (Thermo Fisher Scientific); Collagenase Type I from Clostridium histolyticum (Gibco). Human dental pulp stem cells (DPSC) were purchased from Lonza; cells were cultured in Dulbecco's modified eagle medium + GlutaMAX (DMEM, Gibco), 10\% v/v fetal bovine serum (FBS), 1\% v/v Hepes, 1X penicillin-streptomycin (Gibco).

Synthesis and Characterization of Gelatin Derivatives. Gelatin derivatives were synthesized by decorating gelatin either by methyltetrazine-amine (tetrazine, $\mathrm{Tz}$ ) or 5-norbornene-2-methylamine (norbornene, $\mathrm{Nb}$ ). ${ }^{44}$ Gelatin was dissolved at $1 \% \mathrm{w} / \mathrm{v}$ in MES buffer $(0.1 \mathrm{M}, \mathrm{pH} 6)$ under stirring at $37^{\circ} \mathrm{C}$. For Tz-derivatives synthesis, Tz, EDC, and NHS were added at a molar ratio of 1:4:2 and stirred at $37{ }^{\circ} \mathrm{C}$ in the gelatin solution for $4 \mathrm{~h}$. For Nb-derivative syntheses, Nb, EDC, and NHS were added at a molar ratio of 1:2:1. After $4 \mathrm{~h}$ under stirring, a 1:1 volume of Milli- $\mathrm{Q}$ water was added and stirred for a further $30 \mathrm{~min}$. Gelatin derivatives with different degrees of modification DOM (i.e., low L, medium M, and high $\mathrm{H}$ ) were 
synthesized by adding different amounts of $\mathrm{Tz}$ and $\mathrm{Nb}$ during the synthesis. In particular, $\mathrm{Tz} \_\mathrm{L}, \mathrm{Tz} \_\mathrm{M}$, and $\mathrm{Tz} \_\mathrm{H}$ gelatin derivatives were synthesized by adding $0.5,1$, and $2 \mathrm{mmol}$ of Tz per g of gelatin, respectively. Similarly, $\mathrm{Nb} \_\mathrm{L}, \mathrm{Nb} \_\mathrm{M}$, and $\mathrm{Nb} \_\mathrm{H}$ gelatin derivatives were synthesized by adding $0.5,1$, and $2 \mathrm{mmol}$ of $\mathrm{Nb}$ per $\mathrm{g}$ of gelatin, respectively. Gelatin derivative solutions were then collected, dialyzed against Milli- $Q$ water ( $3.5 \mathrm{kDa}$ molecular weight cutoff) for 4 days, filtered, and freeze-dried.

The degree of modification (DOM) of gelatin derivatives was determined by ${ }^{1} \mathrm{H}$ NMR spectroscopy. Unmodified gelatin and gelatin derivatives were dissolved $3 \% \mathrm{w} / \mathrm{v}$ in deuterium oxide, with $0.05 \mathrm{wt} \%$ TMSP as internal standard. NMR spectra were obtained by Bruker Avance $500 \mathrm{MHz}$ spectrometer at $37{ }^{\circ} \mathrm{C}, 256$ scans, 5 s delay, and analyzed by MestReNova software. The DOM, defined as the molar amount of molecules (i.e., $\mathrm{Tz}$ or $\mathrm{Nb}$ ) per $\mathrm{g}$ of gelatin, was calculated as eq $1^{45}$

$$
\mathrm{DOM}=\frac{\int \text { molecule }}{\int \mathrm{TMSP}} \times \frac{9 \mathrm{H}}{2 \mathrm{H}} \times \frac{n(\mathrm{TMSP})[\mathrm{mmol}]}{m(\text { gelatin })(\mathrm{g})}
$$

Where, $\int$ TMPS is the internal reference signal $(0 \mathrm{ppm} /$ integrating for 9 protons); /molecule is the signal detected for $\mathrm{Tz}(8.5-8 \mathrm{ppm} /$ integrating for 2 protons) in Tz-derivatives or the signal detected for $\mathrm{Nb}$ (6.3-5.9 ppm/integrating for 2 protons) in $\mathrm{Nb}$-derivatives; and $n$ (TMPS) and $m$ (gelatin) are the moles of TMPS and the mass of gelatin in the tested sample, respectively. The DOM was used to calculate the percentage DOM as percentage ratio of $\mathrm{Tz}$ or $\mathrm{Nb}$ measured in the sample to the total amount of carboxyl groups in gelatin (i.e., percentage of carboxylic groups successfully functionalized with $\mathrm{Tz}$ or $\mathrm{Nb}$ ).

Preparation of Gelatin Hydrogels. Gelatin hydrogels (GEL) were prepared at a fixed concentration of $8 \% \mathrm{w} / \mathrm{v}$, by mixing $\mathrm{Tz}$ - and $\mathrm{Nb}$-gelatin derivative solutions to allow the hydrogel network formation by inverse electron demand Diels-Alder reaction. Different hydrogel formulations $(n=9)$ were prepared by varying (1) the DOM of the gelatin derivatives mixed to form the hydrogels (i.e., L, $\mathrm{M}$, or $\mathrm{H}$ ) and (2) the molar ratio $\mathrm{R}$ of $\mathrm{Tz} / \mathrm{Nb}$ in the prepared hydrogels (i.e., $R 05, R 1$, and $R 2$ ). In particular (1), Tz_L and Nb_L derivatives were mixed to obtain GEL $L$ hydrogels; $\mathrm{Tz} M$ and $\mathrm{Nb} M$ were mixed to obtain GEL $M$ hydrogels; $\mathrm{Tz} \mathrm{H}$ and $\mathrm{Nb} \mathrm{H}$ were mixed to obtain GEL $\mathrm{H}$ hydrogels. Moreover $(2)$, the gelatin derivative solutions were mixed at different relative volumes to achieve different molar ratios $\mathrm{R}$ of $\mathrm{Tz} / \mathrm{Nb}$ in the prepared hydrogels. The considered ratios were $R 05, R 1$, and $R 2$ for $\mathrm{Tz} / \mathrm{Nb}$ molar ratios equal to $0.5,1$ and 2, respectively. For instance, GEL_L_R05 was prepared by mixing $\mathrm{Tz} \mathrm{L}$ and $\mathrm{Nb} \mathrm{L}$ solutions at volumes calculated to achieve a ratio $R$ of $\overline{\mathrm{T}} \mathrm{z} / \mathrm{Nb}$ equal to 0.5 in the final hydrogel. The prepared hydrogel formulations are summarized in Table 1.

Physico-mechanical Characterization of Gelatin Hydrogels. The evolution of the rheological properties of hydrogels after mixing the gelatin derivative solutions was measured by rheological tests (Anton Paar MCR302) to investigate their cross-linking kinetic. Tests ( $n=3$ per hydrogel formulation) were run by parallel plate geometry (diameter $\varnothing=25 \mathrm{~mm}$ ) at $37^{\circ} \mathrm{C}, 1 \mathrm{~Hz}$ frequency, and $1 \%$ oscillatory strain. ${ }^{44}$ The storage modulus $\left(G^{\prime}\right)$ and loss modulus $\left(G^{\prime \prime}\right)$ were recorded for $2 \mathrm{~h}$ from the moment the gelatin derivative solutions were mixed and loaded on the rheometer plate. The time to $50 \%$ plateau, $t_{50 \% \text { plateau }}$ was calculated as the time required to reach $50 \%$ of the $G^{\prime}$ values at the end of the each test. ${ }^{44}$

Disk-shaped hydrogel samples were prepared by casting mixed gelatin derivative solutions into PDMS molds, prepared by replica molding of Tough $2000 \mathrm{~V} 1$ resin molds printed by Form3 printer (Formlabs). After mixing and casting the precursors, molds were lodged in Petri dishes and stored at $37^{\circ} \mathrm{C}$ in humidified incubators for $2 \mathrm{~h}$ to allow hydrogels cross-linking and avoid water evaporation before removal from the molds and further characterization.

The swelling and weight variation of the cross-linked gelatin hydrogels was investigated in PBS $(0.01 \% \mathrm{w} / \mathrm{v}$ sodium azide, used as bacteriostatic agent). Freeze-dried samples $(n=4)$ were weighted $\left(w_{0}\right)$, lodged in 24-multiwell tissue culture polystyrene (TCPS),
Table 1. Gelatin Hydrogel Formulations Prepared by Bioorthogonal Cross-Linking between Gelatin Derivatives Modified with Tetrazine $(\mathrm{Tz})$ and Norbornene $(\mathrm{Nb})^{a}$

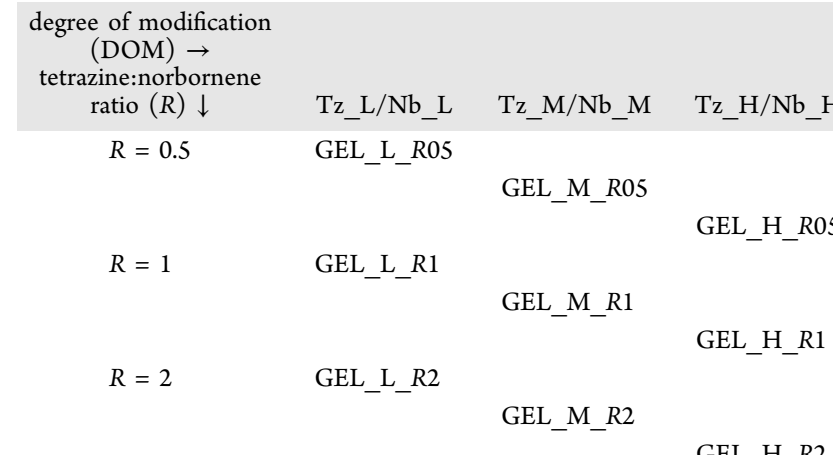

${ }^{a}$ Samples were obtained by mixing gelatin derivatives with different degrees of modification DOM (low, L; medium, M; and high, H) at different $\mathrm{Tz} / \mathrm{Nb}$ ratios $R(0.5,1$, and 2$)$.

immersed in $1.5 \mathrm{~mL}$ PBS, stored at $37{ }^{\circ} \mathrm{C}$ and weighted at established time points $\left(w_{\mathrm{t}}\right)$ up to 14 days. The percentage weight variation $\Delta w$ [\%] was calculated, at each time point $t$, as follows (eq 2):

$$
\Delta w[\%]=\frac{w_{t}-w_{0}}{w_{0}} \times 100
$$

The compressive mechanical properties of swollen cross-linked hydrogels $(n=4)$ were tested by Instron 5900R ( $10 \mathrm{~N}$ load cell). Swollen hydrogels were compressed by one hysteresis cycle by loading at $5 \% \mathrm{~min}^{-1}(0.005 \mathrm{~N}$ preload) and subsequent unloading at $5 \%$ $\mathrm{min}^{-1}$. From the obtained stress-strain $(\sigma-\varepsilon)$ curves, the elastic modulus $E$ (calculated as the slope of the linear interpolation in the $0-5 \%$ strain range during loading, $R^{2}>0.9$ ), the maximum stress $\sigma_{\max }$ (calculated as the stress at $30 \%$ strain) and the residual deformation $\varepsilon_{\text {res }}$ (calculated as the residual deformation at the end of the unloading phase) were considered. ${ }^{46}$

In Vitro 3D Cell Culture. Human dental pulp stem cells (DPSC) were grown in culture medium and used for the tests between passage P3-P5. Cell-laden hydrogels $\left(1 \times 10^{6}\right.$ cells $\left.\mathrm{mL}^{-1}\right)$ were prepared by mixing the gelatin derivative solutions (sterilized by filtration, $\varnothing=$ $0.22 \mu \mathrm{m})$ and by adding the cell suspension. After cross-linking, cellladen hydrogels were lodged in 24-multiwell TCPS, $800 \mu \mathrm{L}$ culture medium was added to each well, and the constructs were cultured in incubator $\left(37^{\circ} \mathrm{C}, 5 \% \mathrm{CO}_{2}\right)$. GEL L and GEL $\mathrm{M}$ hydrogels were considered for the biological in vitro characterization (i.e., GEL L R05, GEL L R1, GEL L R2, GEL M R05, GEL M_ $\bar{R} 1$, and GEL $\bar{M}_{-} \bar{R} 2$; see Results).

The cytocompatibility of the gelatin hydrogels and cross-linking reaction was evaluated after 1 day of culture by live/dead staining. Samples $(n=3)$ were washed twice with Hanks' balanced salt solution (HBSS), immersed in the live/dead staining solution (calcein/AM 1 $\mu \mathrm{M}$, propidium iodide $5 \mu \mathrm{M}$ ) and incubated for $40 \mathrm{~min}$. Samples were washed twice with HBSS and imaged by confocal microscope (scanned thickness $=120 \mu \mathrm{m}$, z-step size $=0.2 \mu \mathrm{m}$; SP8 Confocal Microscope, Leica) to visualize viable and dead cells (i.e., green and red, respectively). Controls were prepared by incubating cell-laden hydrogels in culture medium with $0.5 \%$ Triton X-100. The percentage number of viable cells was calculated ( $n=3$ images for each sample, ImageJ software) as percentage ratio of the number of viable cells (green cells) to the total number of cells (green cells + red cells).

The metabolic activity of cells cultured in the 3D hydrogels $(n=4)$ was measured up to 14 days of culture by AlamarBlue assay. At established time points, samples were transferred to new multiwell TCPS and the culture medium was replaced with the same volume of $10 \%$ AlamarBlue solution in culture medium. Samples were incubated for $4 \mathrm{~h}$, protected from light. Then, $100 \mu \mathrm{L}$ were collected from each sample in triplicates and fluorescence was read (Clariostar Plus Microplate Reader, $\lambda_{\text {excitation }}=560 \mathrm{~nm}$ and $\left.\lambda_{\text {emission }}=590 \mathrm{~nm}\right)$. Gelatin 
hydrogels without cells were also tested, as controls, to remove background fluorescence signal. After the test, samples were washed three times in HBSS and cultured until the following time point.

Cells cultured in the hydrogels were stained by rhodamine phalloidin/Hoechst for F-Actin and nuclei, respectively, after 2 weeks of culture. Samples were washed with PBS, fixed by Image-iT Fixative Solution (4\% formaldehyde, methanol-free) and permeabilized in $0.1 \%$ Triton X-100 solution. Here, $1 \% \mathrm{v} / \mathrm{v}$ BSA was used as a blocking solution. Then, rhodamine phalloidin/Hoechst staining was performed, and cells were distributed in the 3D hydrogel visualized by confocal microscopy (scanned thickness $=80 \mu \mathrm{m}$, z-step size $=0.5$ $\mu \mathrm{m}$; SP8 Confocal Microscope, Leica).

Hydrogel in vitro biodegradation was investigated by an enzymatic assay. Dry samples ( $n=3$ per time point) were immersed in $1 \mathrm{U} \mathrm{mL}^{-1}$ collagenase solution and incubated at $37^{\circ} \mathrm{C} .{ }^{46,47}$ At established time points, samples were removed from the collagenase solution, washed three times in distilled water, freeze-dried, and weighted. The degradation was then calculated by measuring the percentage solid residual weight of the hydrogels as (eq 3)

$$
\text { residual weight [\%] }=\frac{w_{\mathrm{f}}}{w_{\mathrm{i}}} \times 100
$$

Where, $w_{\mathrm{i}}$ and $w_{\mathrm{f}}$ are the dry weights of the hydrogel before and after immersion in enzymatic solution.

Adhesion Tests between Hydrogels. Adhesion between hydrogels was investigated to test their potential use as in vitro compartmentalized coculture systems. We prepared hydrogels with excess $\mathrm{Nb}$ (i.e., R05) and $\mathrm{Tz}$ (i.e., $\mathrm{R} 2$ ) and placed them one on top of each other without compression to facilitate bioorthogonal crosslinking between the excess moieties in the hydrogels (i.e., $R 05 \_R 2$ sample). As controls, we also repeated this experiment with hydrogels prepared with equimolar $\mathrm{Tz} / \mathrm{Nb}$ ratio (i.e., $R 1$ with no excess of $\mathrm{Tz}$ or $\mathrm{Nb}, R 1 R 1)$ and with hydrogels cross-linked with glutaraldehyde (GTA_GTA), ${ }^{48}$ as shown in Figure 7 . The hydrogels were placed in contact for $2 \mathrm{~h}$, and then, the coupled hydrogels were immersed in fluid and stored at $37^{\circ} \mathrm{C}$. After 14 days of culture, coupled hydrogels were handled to qualitatively check their adhesion and the possibility of performing routine cell culture operations without detaching the hydrogels (e.g., culture medium change, sample transfer between TCPS). The adhesion between hydrogels was quantitatively evaluated by tensile tests. ${ }^{49}$ Coupled hydrogels $(n=3$ per combination) were glued to glass coverslips that were fixed to the plate and the upper parallel plate of a rheometer (Kinexus Ultra+, Netzsch). A gap speed of $0.1 \mathrm{~mm} \mathrm{~s}^{-1}$ was applied until hydrogel detached $(0.02 \mathrm{~N}$ preload). The tensile strength TS was calculated from the force-displacement curves as (eq 4)

$$
\mathrm{TS}=\frac{F_{\max }}{A}
$$

Where, $F_{\max }$ is the maximum force detected before hydrogels detachment and $A$ is the contact area between the hydrogels.

Statistical Analysis. Data are shown as mean \pm standard deviation. Statistical analysis was performed by Prism-GraphPad software. Data normal distribution was checked by Shapiro-Wilk test. Differences between data groups were investigated by one-way ANOVA, with Tukey's multiple comparison. Statistical significance between data groups was set for $p<0.05$.

\section{RESULTS}

Characterization of Tetrazine- and NorborneneGelatin Derivatives. Gelatin derivatives functionalized by $\mathrm{Tz}$ and $\mathrm{Nb}$ were successfully synthesized via EDC-NHS chemistry by utilizing $\mathrm{Tz}$ and $\mathrm{Nb}$ amines and the carboxylic groups of the gelatin polymer. The presence of $\mathrm{Tz}$ and $\mathrm{Nb}$ bound to gelatin is demonstrated by the appearance of characteristic peaks of $\mathrm{Tz}$ and $\mathrm{Nb}$ in the ${ }^{1} \mathrm{H}$ NMR spectra at 8.5-8 ppm (Figure 1A) and 6.3-5.9 ppm (Figure 1B), respectively, compared to unmodified gelatin. The degree of
(A)

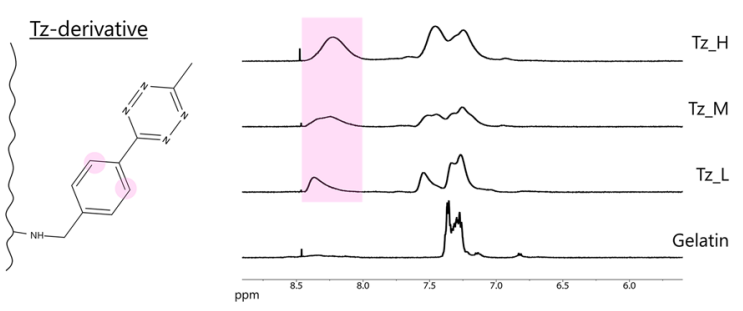

(B)

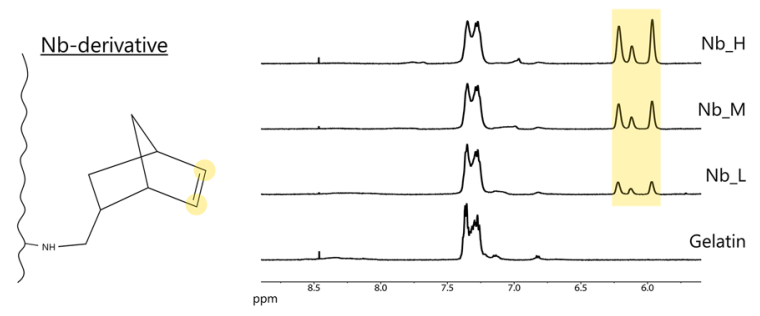

(C)
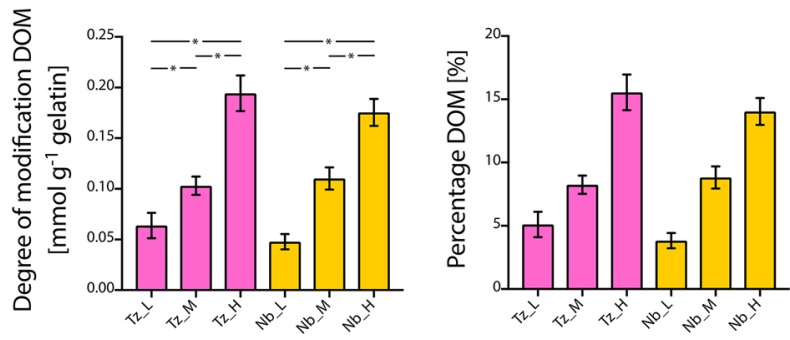

Figure 1. Synthesis of gelatin derivatives modified by tetrazine $(\mathrm{Tz})$ and norbornene $(\mathrm{Nb})$. Representative ${ }^{1} \mathrm{H}$ NMR spectra of $(\mathrm{A}) \mathrm{Tz}$ derivatives modified with different molarities of $\mathrm{Tz}\left(\mathrm{Tz} \_\mathrm{L}, \mathrm{Tz} \_\mathrm{M}\right.$, and $\mathrm{Tz}_{-} \mathrm{H}$ ) and of (B) Nb-derivatives modified with different molarities of $\mathrm{Nb}\left(\mathrm{Nb} \_\mathrm{L}, \mathrm{Nb} \_\mathrm{M}\right.$, and $\left.\mathrm{Nb} \_\mathrm{H}\right)$, compared to unmodified gelatin. (C) Quantification of the degree of modification $\mathrm{DOM}$ (i.e., moles of $\mathrm{Tz}$ or $\mathrm{Nb}$ per $\mathrm{g}$ of gelatin), left, and percentage $\mathrm{DOM}$, right $(n=3$ independent syntheses; mean \pm standard deviation, $\left.*_{p}<0.05\right)$.

modification (DOM) was varied by changing the amount of $\mathrm{Tz}$ and $\mathrm{Nb}$ added during the derivatives synthesis (Figure $1 \mathrm{C}$, left). An increased amount of $\mathrm{Tz}$ lead to an increased DOM from $0.06 \pm 0.01 \mathrm{mmol}$ per $\mathrm{g}$ of gelatin derivative for $\mathrm{Tz} \_\mathrm{L}$, to $0.10 \pm 0.01 \mathrm{mmol}$ per $\mathrm{g}$ of gelatin for $\mathrm{Tz} \mathrm{M}$, to $0.19 \pm 0.02$ mmol per $g$ of gelatin for Tz_H $(p<\overline{0.05}$ comparing Tzderivatives). Similarly, the $\mathrm{DOM}$ of $\mathrm{Nb}$-derivatives was controlled by varying the amount of $\mathrm{Nb}$ added during the synthesis, achieving DOM ranging from $0.05 \pm 0.01 \mathrm{mmol}$ per $\mathrm{g}$ of gelatin for $\mathrm{Nb} \_\mathrm{L}$, to $0.11 \pm 0.01 \mathrm{mmol}$ per $\mathrm{g}$ of gelatin for $\mathrm{Nb} \_\mathrm{M}$, to $0.17 \pm \overline{0} .01 \mathrm{mmol}$ per $\mathrm{g}$ of gelatin for $\mathrm{Nb} \_\mathrm{H}(p<$ $0.0 \overline{5}$ comparing $\mathrm{Nb}$-derivatives). The percentage $\mathrm{D} \overline{\mathrm{O} M}$ was tuned between 5 and $15 \%$ by increasing the amount of $\mathrm{Tz}$ or $\mathrm{Nb}$ added during the synthesis (Figure $1 \mathrm{C}$, right).

The obtained gelatin derivatives were characterized by absent, or reduced, gelation temperature $T_{\text {sol-gel }}$ (Figure S1). Temperature sweep tests showed that unmodified gelatin is characterized by a $T_{\text {sol-gel }}$ of $23.8 \pm 0.3{ }^{\circ} \mathrm{C}$, identified as the intersection of the storage modulus $\left(G^{\prime}\right)$ and loss modulus $\left(G^{\prime \prime}\right)$ curves by decreasing temperature. No gelation temperatures for the gelatin derivatives $\left(\mathrm{Tz} \_\mathrm{L}, \mathrm{Tz} \_\mathrm{M}, \mathrm{Tz} \_\mathrm{H}, \mathrm{Nb} \_\mathrm{M}\right.$, $\left.\mathrm{Nb} \_\mathrm{H}\right)$ or reduced gelation temperatures $\left(\overline{\mathrm{Nb}} \_\mathrm{L}_{\mathrm{L}}, \overline{\mathrm{T}}_{\text {sol-gel }}<10\right.$ $\left.{ }^{\circ} \mathrm{C}\right)$ were identified.

Morphological, Physical, and Mechanical Characterization of Gelatin Hydrogels. Disk-shaped gelatin hydrogels were prepared by mixing $\mathrm{Tz}$ and $\mathrm{Nb}$ gelatin derivatives at $8 \%$ 
Tetrazine/Norbornene Ratio R $(0.5,1,2)$
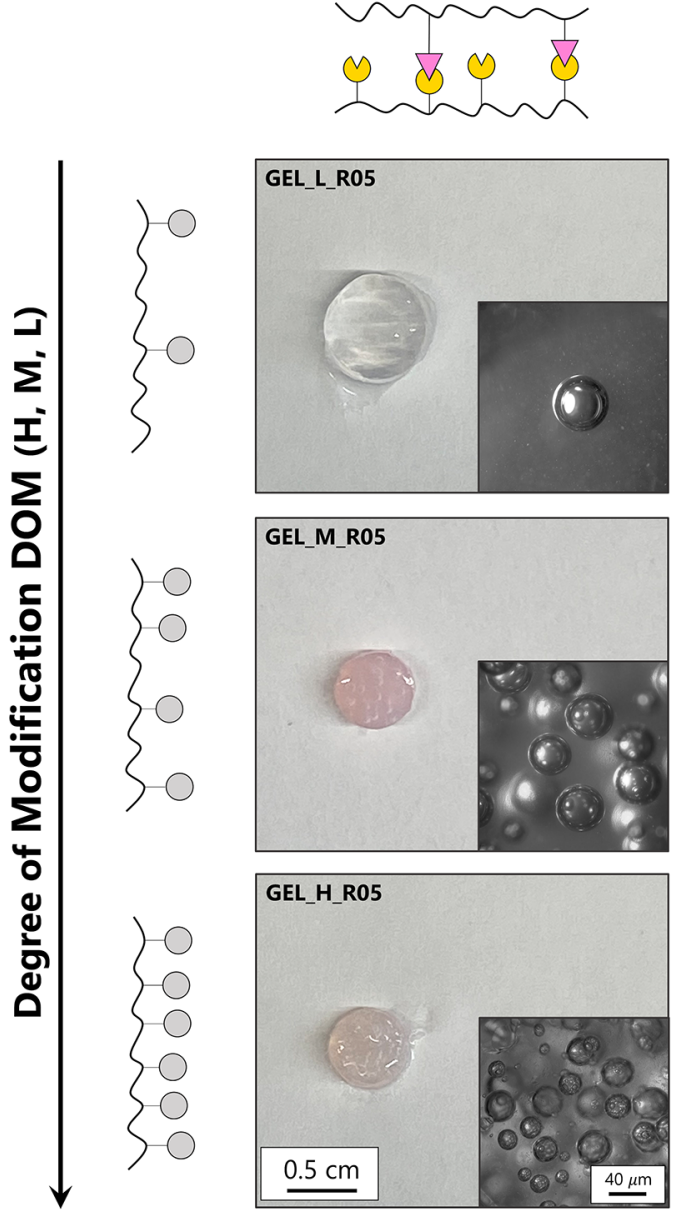
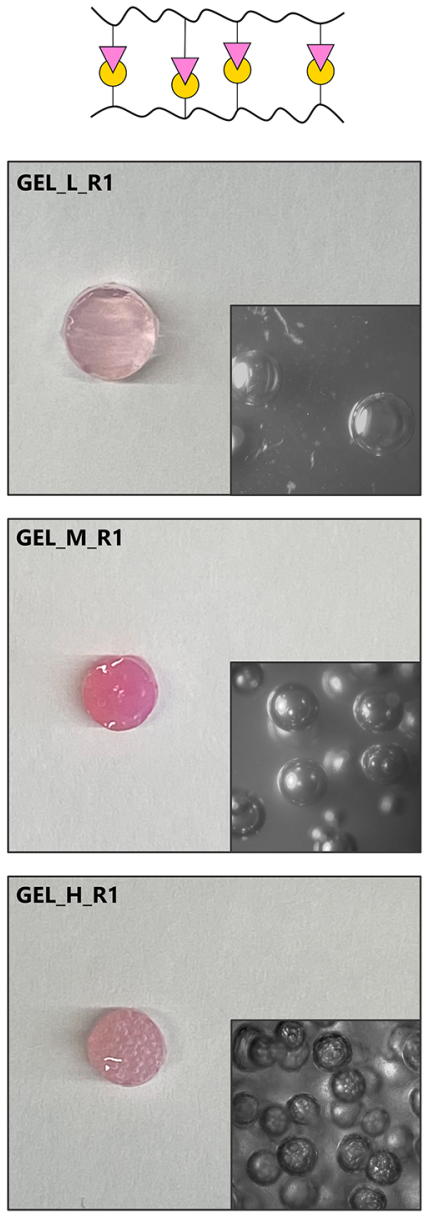
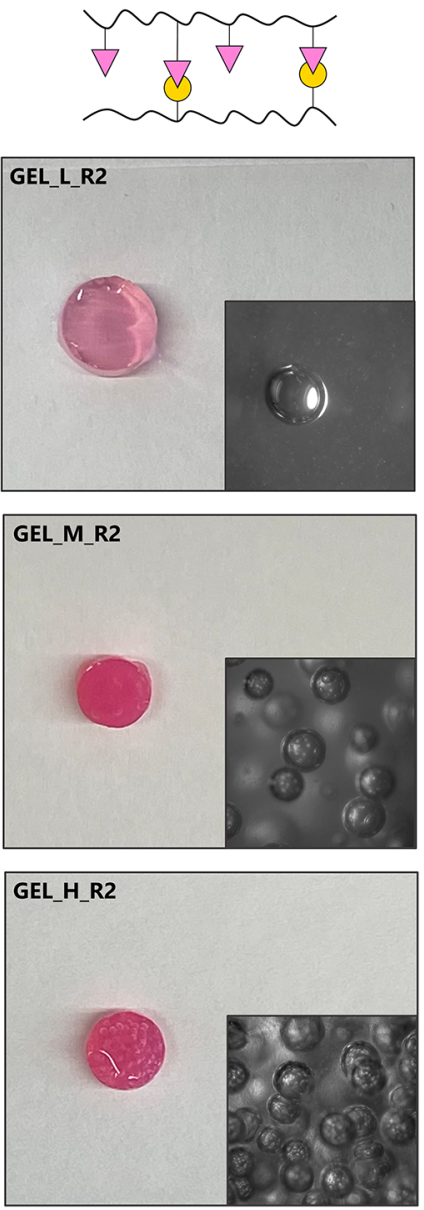

$\checkmark$ Gelatin $\nabla$ Tetrazine
Tetrazine/Norbornene $\circlearrowleft$ Norbornene

Figure 2. Gelatin hydrogels prepared by bioorthogonal cross-linking between tetrazine $(\mathrm{Tz})$ and norbornene $(\mathrm{Nb})$. Hydrogels were prepared by varying the degree of modification DOM (i.e., low L, medium $\mathrm{M}$, and high $\mathrm{H}$, from top to bottom) and the Tz/ $\mathrm{Nb}$ ratio $R$ in the hydrogel (i.e., $R=$ $0.5,1$, and 2, from left to right). Macroscopic appearance of the prepared hydrogels after $24 \mathrm{~h}$ of swelling (scale bar $=0.5 \mathrm{~cm})$ and representative microscopy images in the insets (scale bar $=40 \mu \mathrm{m}$ ).

w/v in PDMS molds (Figure 2). Gelatin derivatives with different $\mathrm{Tz}$ and $\mathrm{Nb} \mathrm{DOM}$ were mixed (Figure 2 from top to bottom, increasing DOM from $\mathrm{L}$, to $\mathrm{M}$, to $\mathrm{H}$ ) at different $\mathrm{Tz}$ / $\mathrm{Nb}$ ratios $R$ (Figure 2 from left to right, increasing $R$ from 0.5 , to 1 , to 2 ). All the prepared hydrogels could be easily removed from the PDMS molds and handled without damage. An increase in the DOM, from $\mathrm{L}$ to $\mathrm{M}$ to $\mathrm{H}$, lead to an evident increase of bubbles inside the cross-linked gelatin hydrogels (Figure 2 insets, from top to bottom). Different ratios $\mathrm{R}$ (Figure 2, from left to right) were used to prepare hydrogels with excess of $\mathrm{Nb}$ ( $R 05$ hydrogels), equimolar $\mathrm{Tz} / \mathrm{Nb}$ ratio ( $R 1$ hydrogels), or excess of $\mathrm{Tz}$ ( $R 2$ hydrogels). The macroscopic appearance of the hydrogels changes by varying the $R$ set for the hydrogel preparation, as a more intense pink color can be observed by increasing the $R$ ratio (Figure 2, from left to right).

The cross-linking kinetics of the gelatin hydrogels was investigated by rheological tests, by recording $G^{\prime}$ and $G^{\prime \prime}$ of the gelatin solutions immediately after mixing the gelatin derivatives to form the hydrogels (Figure $3 \mathrm{~A}$ ). The $G^{\prime}$ of the hydrogels starts increasing immediately after mixing the gelatin derivatives solutions (Figure 3Ai), for all the tested formulations. We considered the time required to reach $50 \%$ of the $G^{\prime}$ values at the end of the tests $\left(t_{50 \% \text { plateau }}\right)$ as an indicator of the speed of the cross-linking reaction and

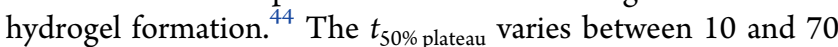
min from the onset of cross-linking (Figure 3Aii), depending on the DOM of the gelatin derivatives used. In fact, if the same $R$ is considered, the quickest cross-linking reaction was observed for hydrogels with high DOM H (i.e., $t_{50 \% \text { plateau }}=$ $10-20 \mathrm{~min})$, the slowest cross-linking reaction was measured for hydrogels with low DOM L (i.e., $t_{50 \% \text { plateau }}=60-70 \mathrm{~min}$ ), and intermediate values were detected for hydrogels with medium DOM M (i.e., $t_{50 \% \text { plateau }}=30-35 \mathrm{~min} ; p<0.05$ comparing the different DOM). For all the tested hydrogel formulations, $G^{\prime}$ exceeded $G^{\prime \prime}$ after mixing the gelatin derivatives, confirming hydrogel network formation with prevalent elastic response $\left(G^{\prime} \gg G^{\prime \prime}\right)$. At the plateau, 
(Ai)

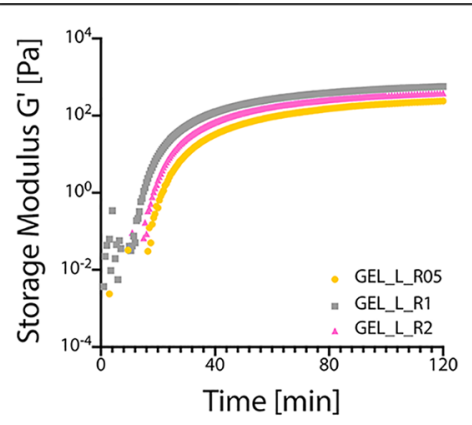

(Aii)

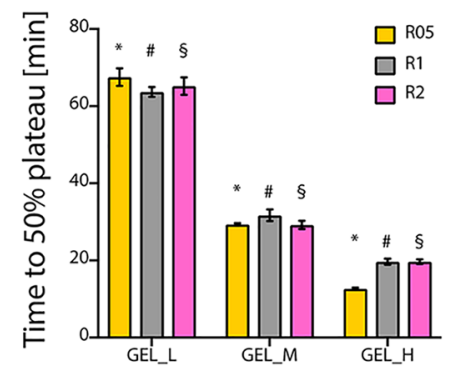

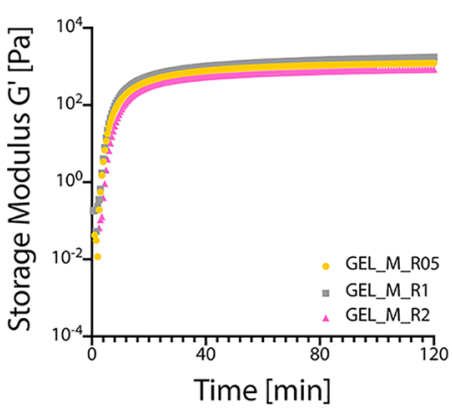
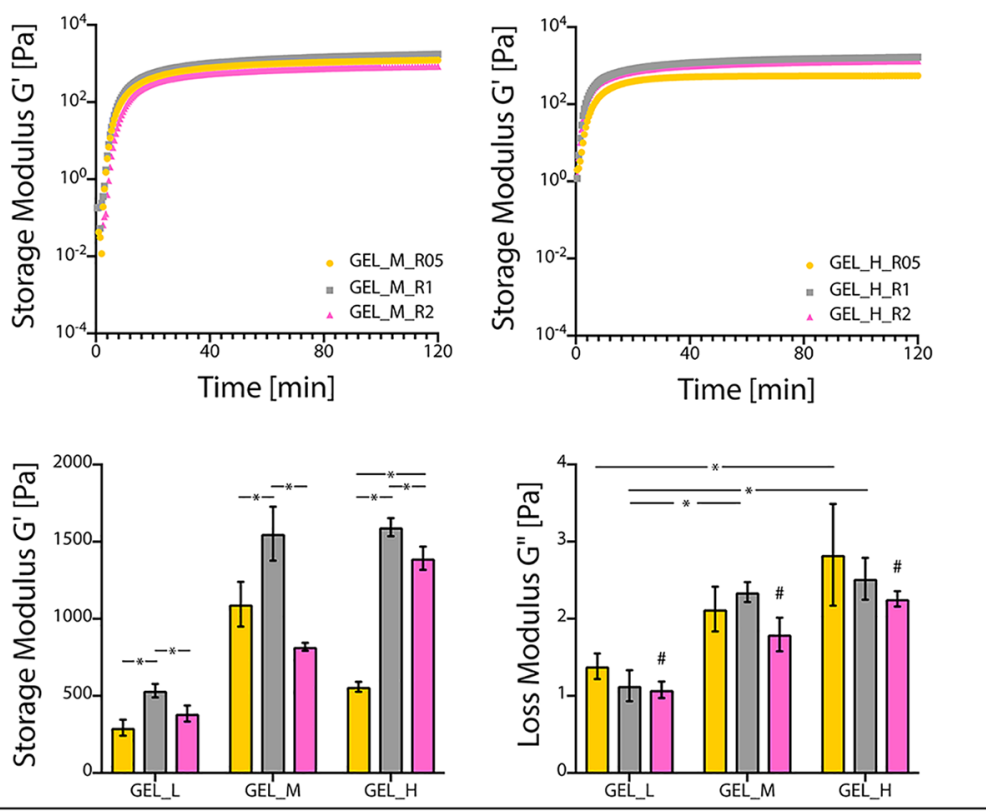

(B)
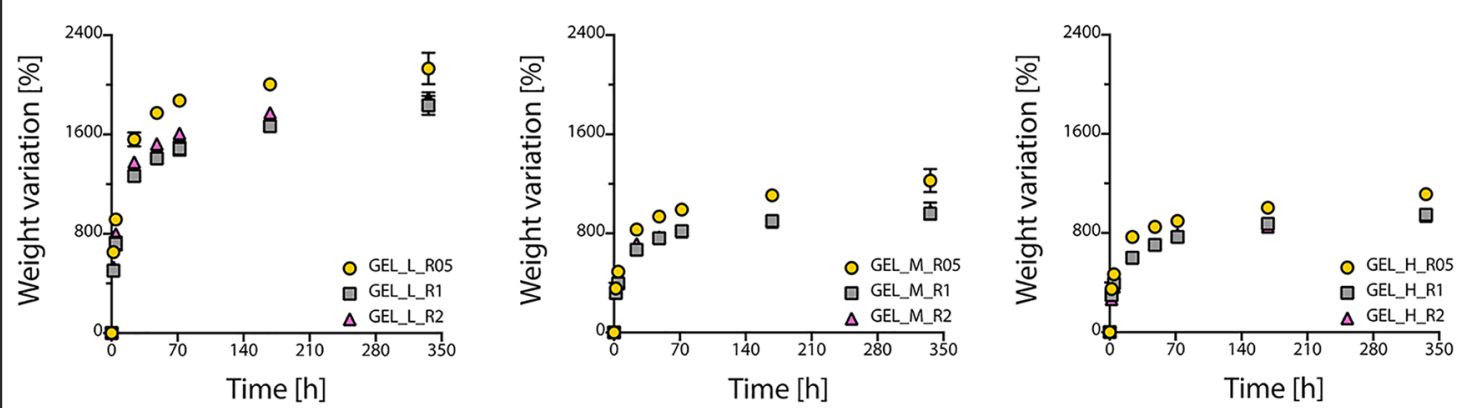

(Ci)

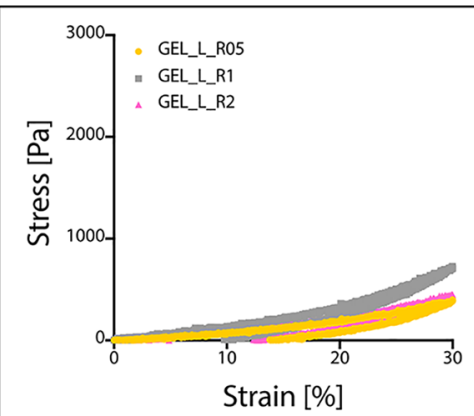

(Cii)
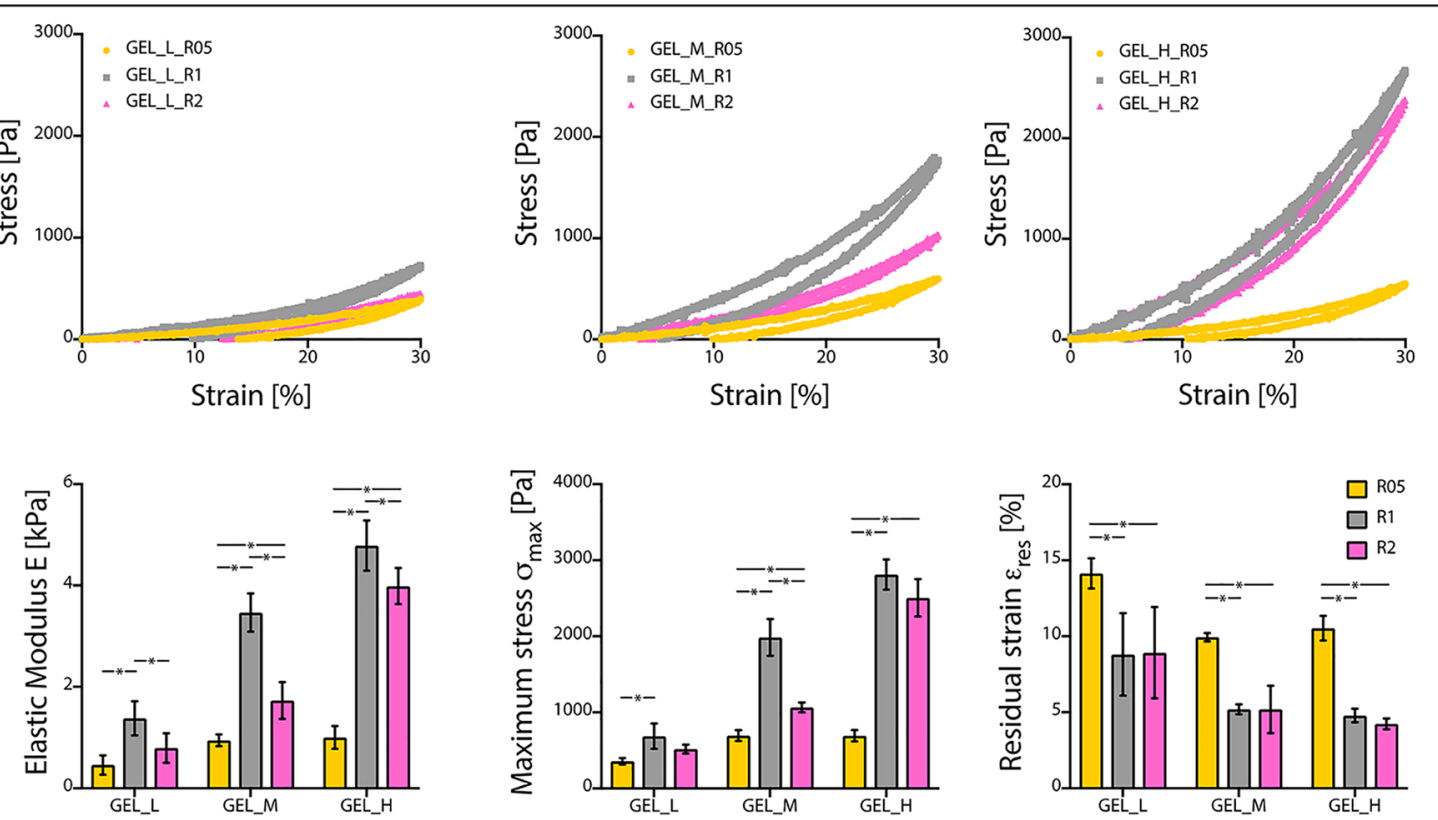

Figure 3. Physico-mechanical characterization of bioorthogonal cross-linked gelatin hydrogels prepared by varying the degree of modification $\mathrm{DOM}$ (i.e., $\mathrm{L}, \mathrm{M}$ and $\mathrm{H}$ ) and the $\mathrm{Tz} / \mathrm{Nb}$ ratio $R$ (i.e., 0.5, 1, and 2). (A) Rheological properties of gelatin hydrogels during cross-linking. (Ai) Representative evolution of the storage modulus, $G^{\prime}$, during hydrogel cross-linking. (Aii) Average storage modulus, $G^{\prime}$, and loss modulus, $G^{\prime \prime}$, at plateau and time required to reach $50 \%$ of the $G^{\prime}$ plateau $(n=3$; mean \pm standard deviation, $* \# \S p<0.05)$. (B) Percentage weight variation of gelatin hydrogels in PBS at $37{ }^{\circ} \mathrm{C}(n=4)$. (C) Compressive mechanical properties of swollen gelatin hydrogels. (Ci) Representative stress-strain curves of hydrogels prepared with different DOM and $R$. (Cii) Mechanical parameters (i.e., elastic modulus $E$, maximum stress $\sigma_{\max }$ and residual deformation $\left.\varepsilon_{\text {res }}\right)$ calculated from the stress-strain curves $(n=4$; mean \pm standard deviation, $* p<0.05)$. 
(A)
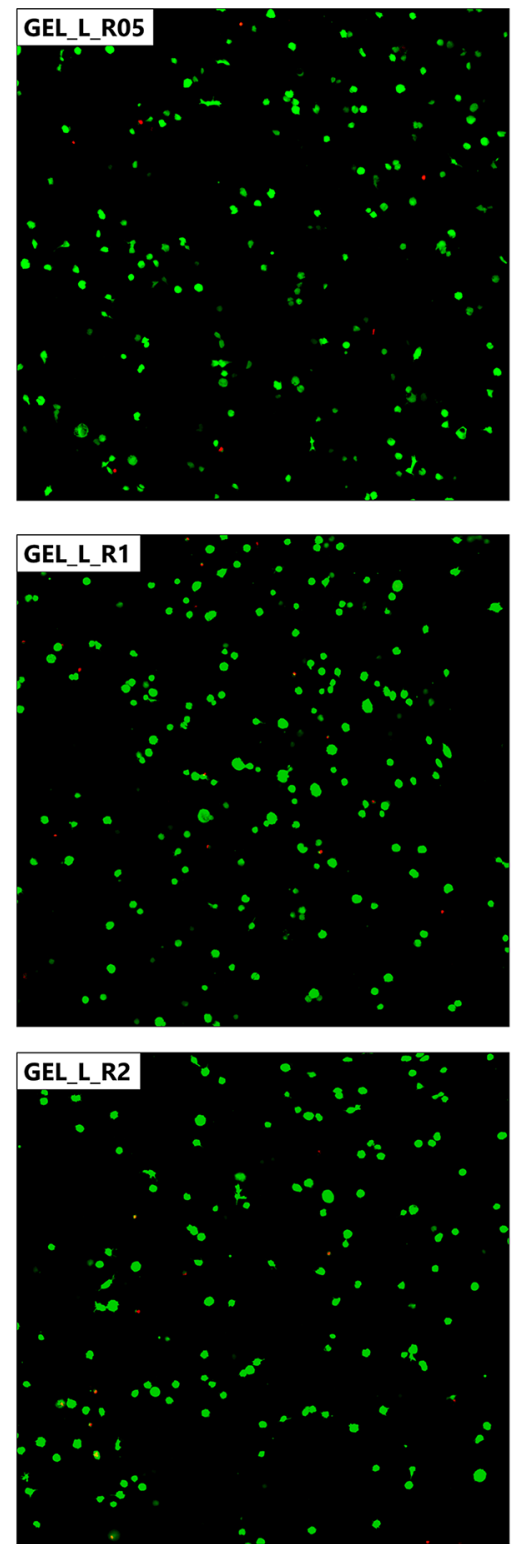

(B)

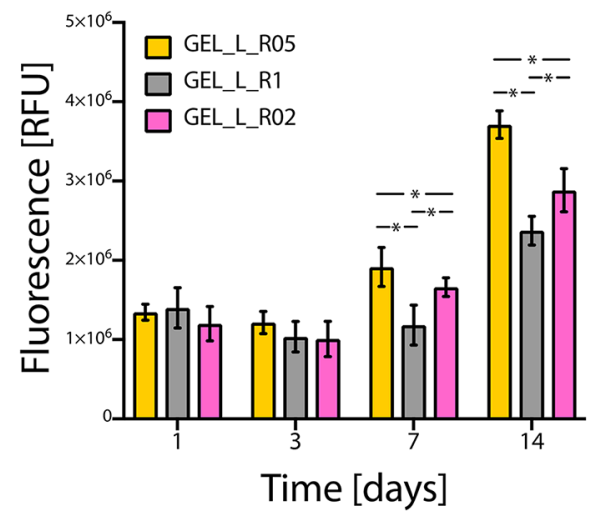

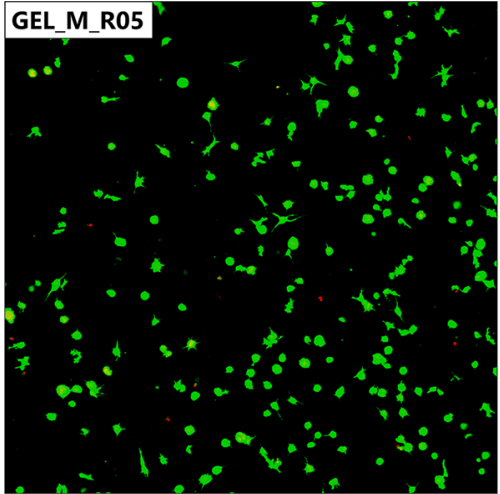
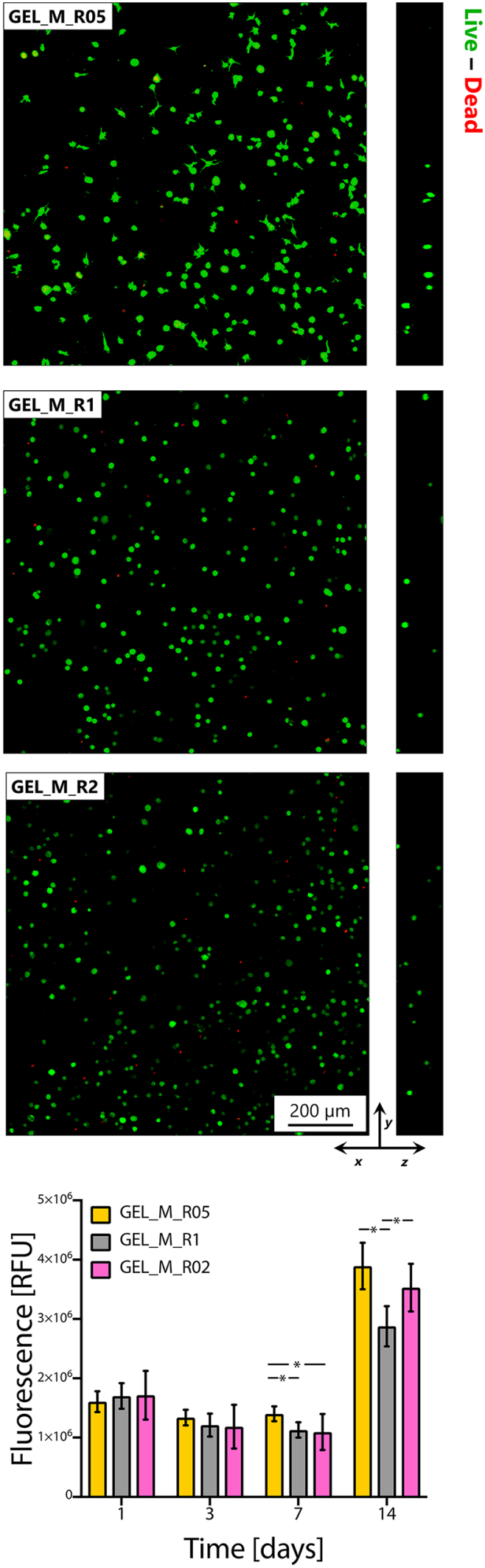

Figure 4. In vitro culture of human DPSCs in 3D gelatin hydrogels. (A) Representative live/dead images showing viable (green) and dead (dead) cells after 1 day of culture. For each image, the top $(X-Y$ plane $)$ and section $(Y-Z$ plane $)$ views are shown $(\mathrm{scale}$ bar $=200 \mu \mathrm{m})$. (B) Metabolic activity of cells cultured in the $3 \mathrm{D}$ hydrogels for up to 14 days, measured by AlamarBlue assay $(n=4$; mean \pm standard deviation, $*<0.05)$.

hydrogels with low DOM L exhibited the lowest $G^{\prime}$ and $G^{\prime \prime}(p$ $<0.05)$. Moreover, if the same DOM is considered, different $G^{\prime}$ values were measured for hydrogels prepared with different
$R$ ratios. In particular, for all the tested DOM, hydrogels prepared with an equivalent $\mathrm{Tz} / \mathrm{Nb}$ ratio $R 1$ were charac- 
terized by higher $G^{\prime}$ values, compared to $R 05$ and $R 2$ hydrogels (i.e., $\mathrm{Tz}$ and $\mathrm{Nb}$ defect, respectively).

After cross-linking, hydrogels were immersed in PBS at 37 ${ }^{\circ} \mathrm{C}$, to investigate their stability and swelling in a physiologicallike aqueous environment. After immersion, hydrogels increased their weight by absorbing water (Figure 3B), and they could be handled for the duration of the test, proving the formation of a hydrogel network capable of fluid absorption. Different weight variations were detected comparing the hydrogel formulations. The percentage weight variation of GEL_L hydrogels after 14 days of culture varied from $2131 \pm$ $126 \%$, to $1834 \pm 76 \%$, to $1889 \pm 51 \%$ for GEL L R05, GEL_L_R1, and GEL_L_R2, respectively. For the medium $\operatorname{DOM} \bar{M}$, the calculated weight variations were $1225 \pm 93 \%$, $960 \pm 23 \%$, and $980 \pm 69 \%$ for GEL_M_R05, GEL_M_R1, and GEL_M_R2, respectively. Finally, for the highest $\overline{D O} \bar{M} H$, the measured weight variation values were $1114 \pm 24 \%, 948 \pm$ $45 \%$, and $936 \pm 19 \%$ for GEL_H_R05, GEL_H_R1, and GEL_H_R2, respectively. Considering the same $\overline{D O} \bar{M}$ used to prepare the hydrogels, hydrogels prepared with a $\mathrm{Tz} / \mathrm{Nb}$ ratio $R 05$ were characterized by a higher increase in weight after 14 days of swelling, compared to hydrogels prepared with $R 1$ and $R 2(p<0.05)$. Moreover, for all the considered ratios $R$, hydrogels prepared with a low DOM L were characterized by a higher weight variation, compared to hydrogels prepared with medium $\mathrm{M}$ and high H DOM $(p<0.05)$. These differences in hydrogel swelling were consistent with the differences between the calculated swelling ratio values (Figure S2A). After 14 days of swelling, hydrogels were dehydrated, and the percentage solid gel fraction after swelling was calculated (Figure S2B). A higher percentage solid fraction was retained in hydrogels with higher DOM (i.e., percentage gel fraction for DOM M and $\mathrm{H}$ $>70 \%$ ), compared to hydrogels with lower DOM (i.e., percentage gel fraction for DOM L $<70 \%$; $p<0.05$ ). Moreover, if the same DOM is considered, hydrogels prepared with $R 05$ were characterized by lower gel fraction values compared to $R 1$ and $R 2$ hydrogels $(p<0.05)$, indicating a higher loss of gelatin during swelling.

The mechanical properties of the gelatin hydrogels were tuned by varying DOM and $R$. Representative stress-strain curves of swollen hydrogels are shown in Figure 3Ci. For all the hydrogels, curves are characterized by an increase in stress during the loading phase (i.e., application of strain) and by a decrease in the stress during the unloading phase (i.e., removal of strain). The loading and unloading stress-strain curves do not overlap, indicating energy loss typical of viscoelastic materials, and residual deformation can be observed at the end of the unloading phase. The elastic modulus $E$ varied approximately by an order of magnitude between 0.5 and 5 $\mathrm{kPa}$, by combining different DOM and $R$. The compressive $E$ values of hydrogels prepared using gelatin derivatives with low DOM L were $0.45 \pm 0.19,1.37 \pm 0.33$, and $0.79 \pm 0.29 \mathrm{kPa}$ for GEL L R05, GEL L $R 1$, and GEL $L R 2$, respectively. When using medium $\overline{\mathrm{D} O M} \mathrm{M}$ gelatin $\overline{\text { derivatives, }} E$ values were $0.94 \pm 0.11,3.46 \pm 0.37$, and $1.72 \pm 0.36 \mathrm{kPa}$ for GEL_M R05, GEL $M R 1$, and GEL $M R 2$, respectively. Finally, for DOM H hydrogels, $E$ values were $1.00 \pm 0.22$, 4.78 \pm 0.49 , and $3.98 \pm 0.35 \mathrm{kPa}$ for GEL_H_R05, GEL_H_R1, and GEL_H_R2, respectively. A higher elastic modulus $\bar{E}$ was measured for hydrogels prepared with a higher DOM and for hydrogels prepared with an equimolar $\mathrm{Tz} / \mathrm{Nb}$ ratio $R$ (i.e., $R 1$ vs $R 05$ and $R 2$ ). In particular, for the $R 05$ ratio the $E$ values of hydrogels prepared with low DOM L were lower than medium
DOM M and high DOM H $(p<0.05)$. For $R 1$ and $R 2$ ratios, the $E$ values of low DOM L hydrogels were lower than medium DOM M and high DOM H hydrogels $(p<0.05)$; moreover, $E$ values of medium DOM M hydrogels were lower than high DOM H $(p<0.05)$. For all the synthesized DOM, $R 1$ hydrogels were characterized by higher E compared to R05 and $R 2$ hydrogels $(p<0.05) . R 2$ hydrogels were characterized by higher E values compared to R05 hydrogels $(p<0.05)$, considering DOM M and $\mathrm{H}$ hydrogels. Similar trends were observed when measuring the maximum stress $\sigma_{\max }$. Higher $E$ values lead to higher $\sigma_{\max }$ at the end of the loading phase, with higher values for hydrogels prepared with higher DOM and equimolar $R$ (i.e., $R 1$ vs $R 05$ and $R 2 ; p<0.05$ ). Finally, residual strain $\varepsilon_{\text {res }}$, an indication of a viscous mechanical response of the prepared hydrogels, was measured for all the formulations, with values varying between 4 and $14 \%$. Hydrogels prepared with low DOM L were characterized by higher $\varepsilon_{\text {res, }}$, indicating a lower recovery of the strain upon unloading. Moreover, hydrogels prepared with the $R 05$ ratio were characterized by higher $\varepsilon_{\text {res }}$ compared to $R 1$ and $R 2$ hydrogels $(p<0.05)$.

In Vitro Biological Characterization and 3D Cell Culture. The in vitro biological characterization was performed on hydrogels prepared from derivatives with low and medium DOM using human DPSC as cell model. Gelatin derivative solutions with high DOM could not to be filtered for sterilization and high DOM H hydrogels were characterized by a prevalent presence of bubbles. The prevalent presence of bubbles might in fact interfere with routine microscopy observation, image acquisition and biological response.

Indirect in vitro cytotoxicity tests confirmed the absence of cytotoxic products from the prepared hydrogels (Figure S3). Cells cultured in medium extracts (i.e., medium previously in contact with gelatin hydrogels) showed percentage viability values higher than $90 \%$, compared to cells culture in culture medium controls (i.e., no contact with hydrogels). Based on the performed indirect in vitro cytotoxicity tests, no cytotoxicity was detected for the culture medium eluates previously put in contact with the hydrogels.

Human DPSCs were added to the gelatin derivatives solutions that were then mixed and cross-linked to form cellladed 3D gelatin hydrogels. After 1 day of culture, live/dead staining was performed to investigate the cytocompatibility of hydrogels. Representative confocal microscopy images of cells cultured in the hydrogels are shown in Figure 4A (viable cells are depicted in green, dead cells in red). For all the tested formulations, cells appear well-distributed in the gelatin hydrogels ( $X-Y$ axes, top view images). Moreover, crosssectional images show that cells are distributed in the 3D structure of the hydrogels ( $Y-Z$ axes, section images). Cell distribution in the 3D structure of the hydrogels is confirmed in the representative $3 \mathrm{D}$ reconstruction of the scanned volume (Video S1). For all the considered hydrogels, the percentage number of viable cells is higher than $85 \%$ (Figure S4), proving the cytocompatibility of the cross-linking reaction and the prepared hydrogels. Moreover, no differences in the percentage number of viable cells were detected comparing the different hydrogel formulations $(p>0.05)$.

After proving the cytocompatibility of the cross-linking procedure and hydrogels, we investigated the metabolic activity of cells cultured in the cross-linked gelatin hydrogels up to 14 days of culture (Figure 4B). The fluorescence signal detected for all the considered time points demonstrated the presence of viable cells inside the hydrogels for the whole 

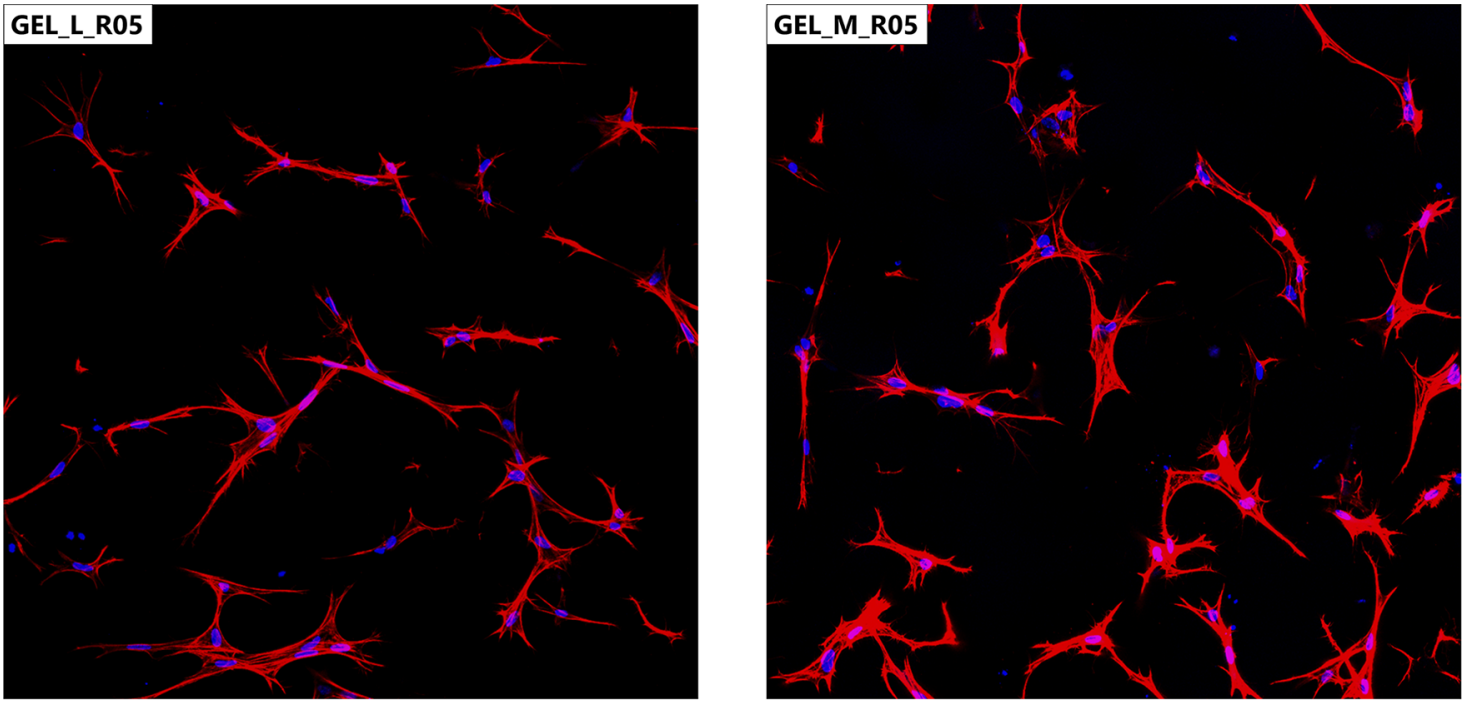

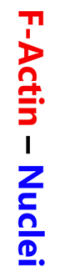
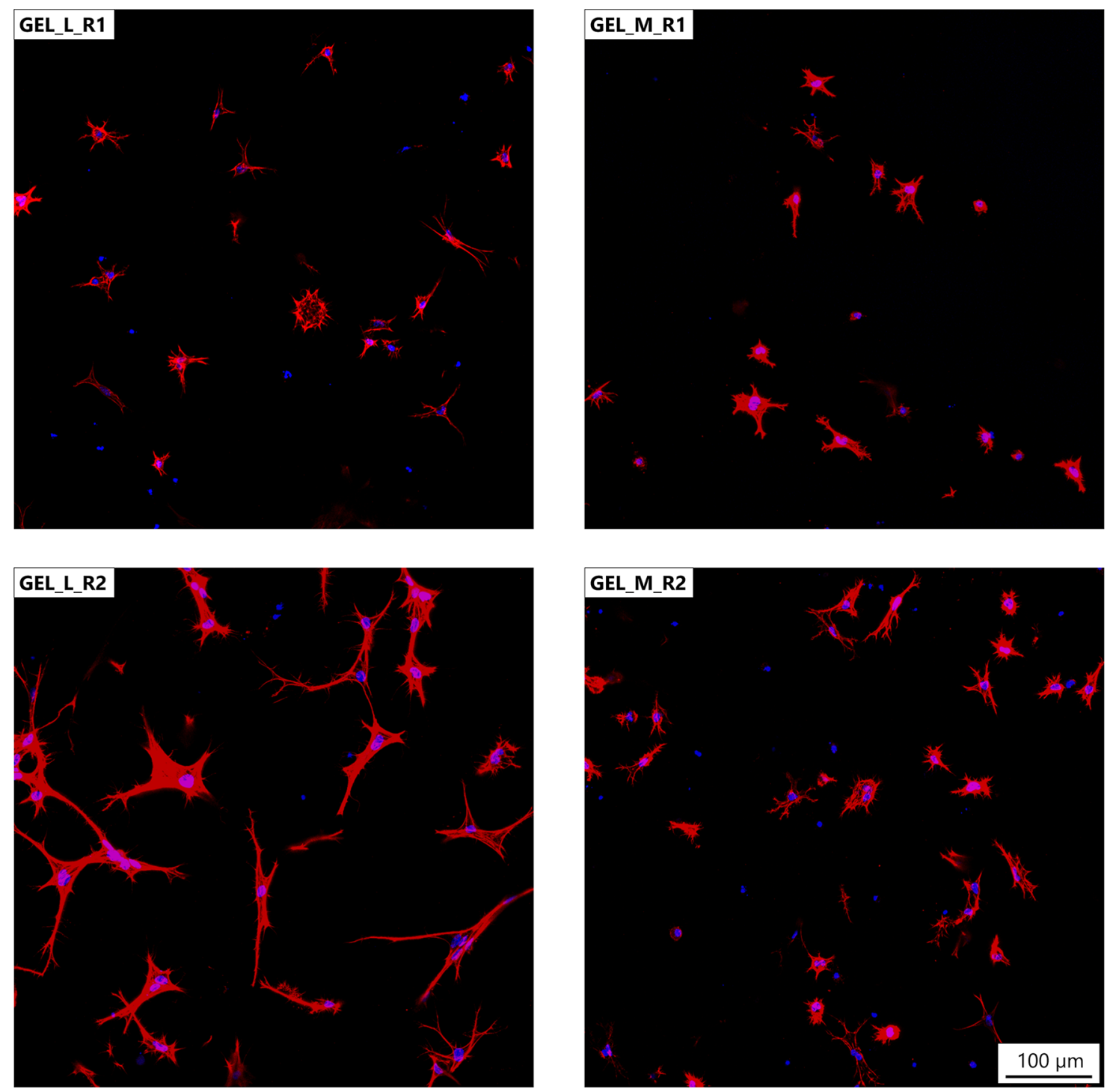

Figure 5. Representative fluorescence images of nuclei (blue), stained by Hoechst, and F-Actin staining (red), stained by rhodamine phalloidin, of human DPSC after 14 days of 3D in vitro culture in gelatin hydrogels (scale bar $=100 \mu \mathrm{m})$.

culture period. Moreover, after 7 and 14 days of culture, we observed different metabolic activities for cells cultured in the different hydrogel formulations. In particular, after 14 days of culture in hydrogels with low DOM L (Figure 4B, left), cells 
cultured in GEL_L_R05 showed higher metabolic activity than cells cultured in GEL_L_R2 hydrogels $(p<0.05)$. Both GEL_L_R05 and GEL_ $\bar{L}-\bar{R} 2$ showed higher values compared to $\overline{G E L} L L \_R 1 \quad(p<\overline{0} . \overline{05})$. Considering cells cultured in hydrogels with medium DOM M (Figure 4B, right), a higher metabolic activity was measured for cells cultured in GEL_M_R05 and GEL_M_R2 hydrogels, compared to GEL_M_R1 $(p<0.05)$. The average percentage increase of metabolic activity was then calculated as percentage ratio of the metabolic activity of cells after 14 days of culture to the metabolic activity after 1 day of culture. The highest increase in metabolic activity over the culture period was measured for cells cultured in GEL_L_R05, GEL_L_R2, and GEL_M_R05 (i.e., approximately $\overline{2} 5 \overline{0} \%$ ). A relatively lower percentage increase was measured for GEL_M_R2 (i.e., approximately $200 \%$ ), and finally, the lowest increase in metabolic activity was measured for GEL L $R 1$ and GEL $M \_R 1$ (i.e., approximately $150 \%)$. Evident differences in cell shape were observed across the different hydrogel samples. After 7 days of culture, microscopy images (Figure S5) showed a clearly elongated shape for cells culture in GEL_L_R05, GEL_L_R2, and GEL_M_R05, compared to the spherical shape characterizing cells cultured in GEL_L_R1, GEL_M_R1, and GEL_M_R2. These observations were further shown by F-actin staining after 14 days of culture (Figure 5). A striking difference can be observed for cells cultured in GEL_L_R05, GEL_L_R2, and GEL M R05, where cells appear spread in the hydrogels with elongate $\bar{d}$ shape characterized by extended actin filaments, compared to cells cultured in GEL_L_R1, GEL_M_R1, and GEL_M_R2, where cells are characterized by a more spherical shape, less elongated and actin filaments distributed around the cell nuclei.

Enzymatic degradability was tested in vitro by immersing hydrogels in collagenase type I solution. All the tested hydrogel formulations were completely degraded (Figure 6). The degradation kinetics were different in time, depending on the $\mathrm{DOM}$ and $\mathrm{Tz} / \mathrm{Nb}$ ratio used to prepare the hydrogels. GEL_L hydrogels degraded faster than GEL_M hydrogels. Moreover,

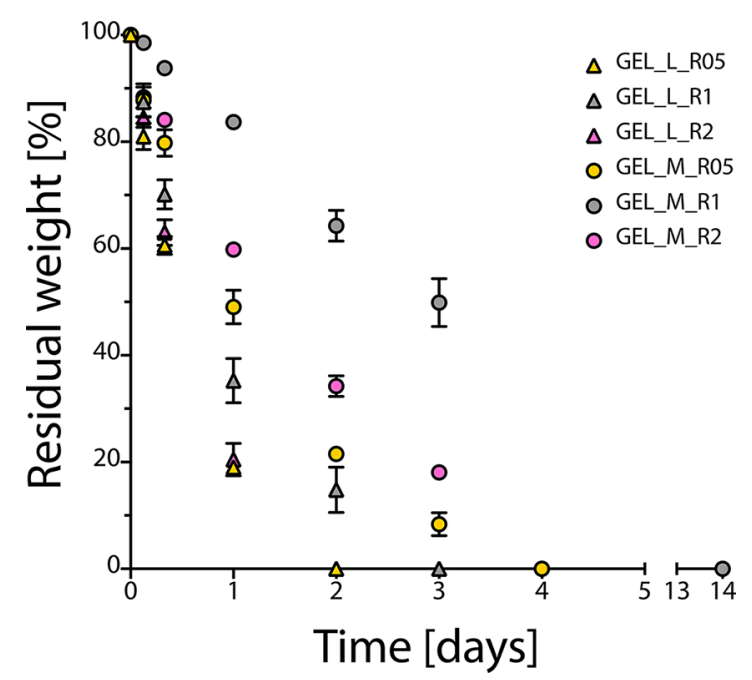

Figure 6. In vitro enzymatic degradation of gelatin hydrogels prepared by different degree of modification DOM (i.e., $\mathrm{L}$ and $\mathrm{M}$ ) and $\mathrm{Tz} / \mathrm{Nb}$ ratio (i.e., $R=0.5,1$, and 2 ), after immersion in collagenase type $\mathrm{I}$ ( 1 $\left.\mathrm{U} \mathrm{mL} \mathrm{m}^{-1}, 37^{\circ} \mathrm{C}\right)$. Data show the residual solid weight of the hydrogels after immersion in enzymatic solution in time $(n=3$; mean \pm standard deviation). if the same DOM is considered, hydrogels prepared with $R 05$ and $R 2$ ratios degraded faster (i.e., 2 and 4 days for GEL_L and GEL_M, respectively) than hydrogels prepared with equimolar $\overline{\mathrm{Tz}} / \mathrm{Nb} R 1$ ratio (i.e., 3 and 14 days, respectively).

Adhesion between Hydrogels. Adhesion between hydrogels was first qualitatively evaluated by handling the hydrogels (Figure 7A, bottom). Samples obtained by adhering $R 05$ and $R 2$ hydrogels could be handled as compact structures and did not detach one from another (Figure 7, center), qualitatively indicating successful adhesion between the two hydrogels. Conversely, immediate detachment between hydrogels was observed when handling samples prepared using $R 1$ hydrogels (Figure 7A, left) and hydrogels cross-linked with glutaraldehyde (Figure 7A, right), suggesting that interhydrogel cross-linking of unreacted $\mathrm{Tz}$ and $\mathrm{Nb}$ moieties is essential in maintaining hydrogel adherence during the culture period. Successful handling of the R05_R2 adhered hydrogels is shown in Videos S2 and S3, compared to the R1_R1 and GTA GTA hydrogels. These qualitative observations were confirmed by tensile adhesion tests (Figure 7B). During the tensile tests, the maximum force was measured prior to adhesive failure (i.e., rupture at the interface between hydrogels in contact). R05 R2 hydrogels were characterized approximately by double the adhesion tensile strength compared to R1_R1 and GTA_GTA hydrogels (Figure 7B, inset graph).

\section{DISCUSSION}

Engineering cytocompatible hydrogels with tunable properties is fundamental for the development of $3 \mathrm{D}$ artificial ECM for tissue engineering and in vitro modeling applications. The selection of the polymer used to prepare the hydrogel and its cross-linking are critical to control the physico-mechanical properties of the hydrogels and achieve biomimetic, cytocompatible hydrogels to guide a desired cell response. ${ }^{1,3}$ In this work, we prepared gelatin derivatives, decorated with either tetrazine $(\mathrm{Tz})$ or norbornene $(\mathrm{Nb})$, to form the polymeric structure of the hydrogel and we prepared the gelatin hydrogels by mixing the derivatives with different degrees of modification and $\mathrm{Tz} / \mathrm{Nb}$ ratios.

Gelatin represents an optimal choice to prepare ECM biomimetic hydrogels. In fact, being a collagen derivative, gelatin possesses unique advantages, compared to other synthetic and natural-derived polymers, including intrinsic presence of cell-adhesive motifs and MMP-sensitive sequences that guarantee cell adhesion and matrix remodeling, fundamental for cell-biomaterial interactions and potential tissue formation/regeneration. ${ }^{13,14}$ Collagen source (e.g., porcine, bovine, fish) and its processing to extract gelatin (e.g., acidic or alkaline for type $\mathrm{A}$ and type $\mathrm{B}$ gelatin, respectively) can heavily influence the properties of the obtained gelatin hydrogels. ${ }^{48,50}$ Thus, we selected type B gelatin as, compared to other gelatin types, it induces minor in vivo inflammatory response, and it exhibits lower immunogenicity and higher cytocompatibility. ${ }^{51,52}$ Moreover, we prepared hydrogels using low-endotoxin gelatin, as controlled endotoxin levels must be guaranteed for potential translation from in vitro studies to in vivo applications, given regulatory constraints and endotoxin-related potential inflammatory effects. ${ }^{53}$

We selected a bioorthogonal cross-linking strategy based on the inverse-electron demand Diels-Alder reaction between $\mathrm{Tz}$ and $\mathrm{Nb}$ to cross-link the gelatin hydrogels we prepared. This 
(A)
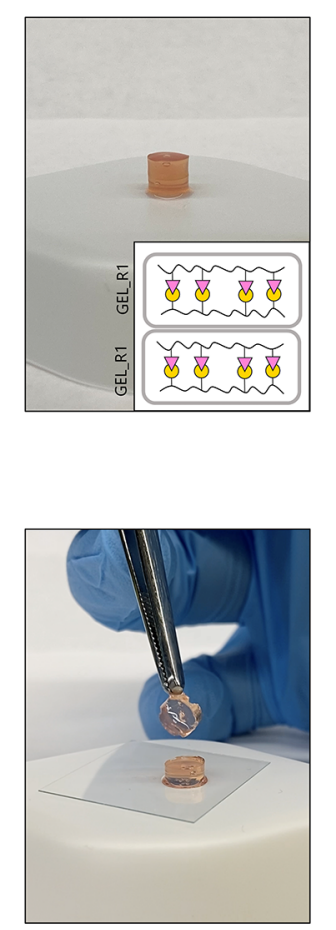

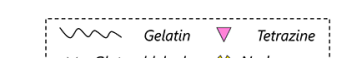

₹ Glutaraldehyde \& Norbornene
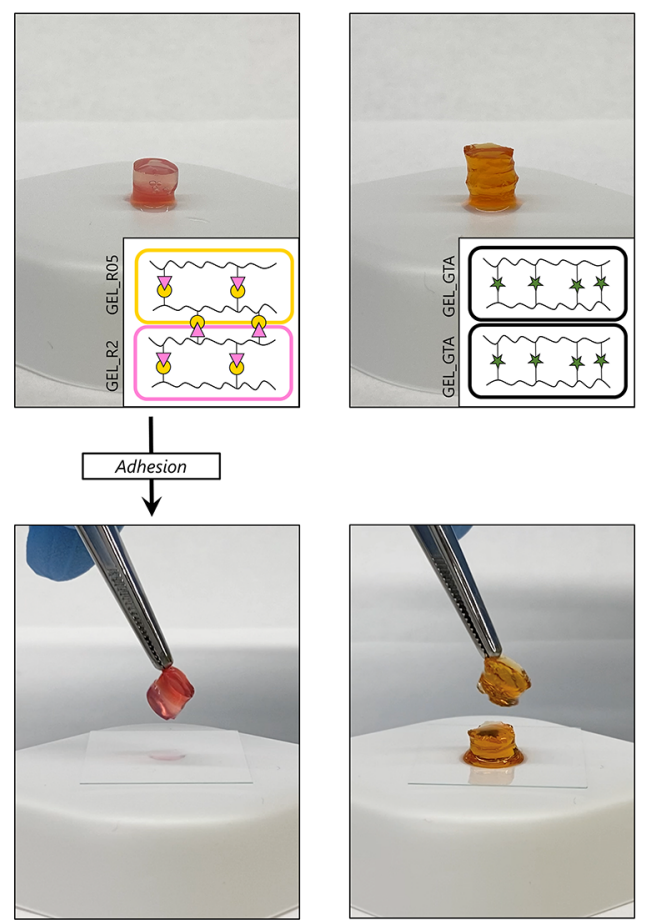

(B)

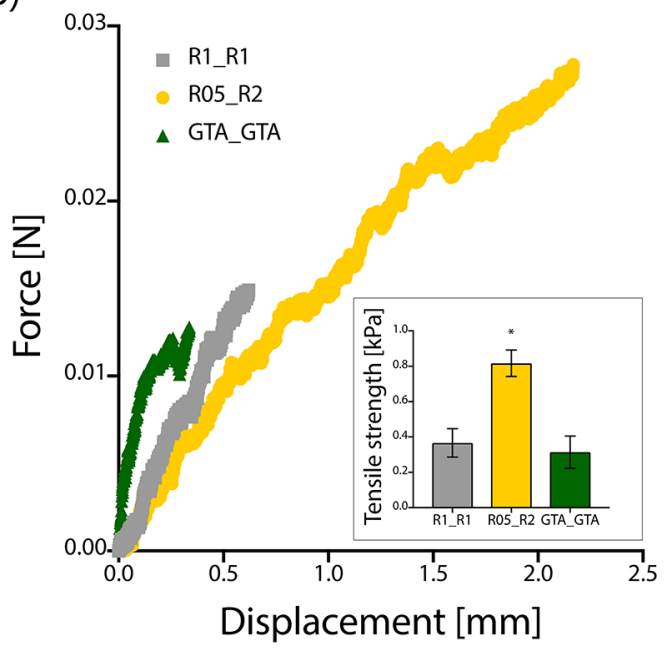

Figure 7. Adhesion between gelatin hydrogels. (A) Gelatin hydrogels (top) were prepared using Tz-Nb bioorthogonal cross-linking ( $R 1$ in contact with $R 1$, on the left, and $R 05$ in contact with $R 2$, center) and glutaraldehyde as cross-linker (right). Excesses of norbornene and tetrazine in $R 05$ and $R 2$ hydrogels, respectively, allowed hydrogels to be handled in contact (bottom, center), while detachment was observed between the control hydrogels (bottom, left, and right). (B) Representative force-displacement curves obtained by adhesion tensile tests on hydrogels placed in contact. Hydrogels coupled included the following: (1) two samples prepared with equimolar Tz/Nb ratio $R 1$ (i.e., $R 1 \_R 1$ ), (2) one sample with $R 05$ with one sample with $R 2$ ratio (i.e., $R 05 \quad R 2$ ), and (3) two samples cross-linked by glutaraldehyde (i.e., GTA GTA). The maximum force reached during the tensile test was used to calculate the adhesive tensile strength (inset graph, $n=3$; mean \pm standard deviation, $* p<0.05$ ).

cross-linking reaction is highly selective, it can occur in mild, cell-friendly conditions, ${ }^{32-34}$ and it does not require that any external energy input and hydrogels can be easily prepared by simply mixing gelatin derivatives functionalized either by $\mathrm{Tz}$ or $\mathrm{Nb}$. To date, the potential of gelatin hydrogels cross-linked by $\mathrm{Tz}-\mathrm{Nb}$ bioorthogonal cross-linking has been investigated in one study, ${ }^{44}$ which described this cross-linking reaction and investigated the influence of polymer concentration and $\mathrm{Tz} /$ $\mathrm{Nb}$ ratio on the properties of the hydrogels and their in vitro and in vivo biological response. Thus, we here further explore the potential of such hydrogels and successfully modulate the properties of the hydrogels by fixing their concentration and varying their cross-linking architecture, by changing the degree of modification (DOM) and the molar ratio $\mathrm{Tz} / \mathrm{Nb}$ in the hydrogels. The use of gelatin as backbone polymer to form the hydrogels allows a better mimicking of the native ECM, thanks to the ECM biomimetic motifs naturally present in gelatin. When other polymers (e.g., alginate, ${ }^{36} \mathrm{PEG}^{35}$ and methylcellulose $)^{38}$ were used to prepare hydrogels by $\mathrm{Tz}-\mathrm{Nb}$ crosslinking, an additional synthesis step was required to artificially introduce ECM biomimetic motifs to improve their biological performance. Conversely, it was previously demonstrated ${ }^{44}$ that gelatin hydrogels modified by $\mathrm{Tz}-\mathrm{Nb}$, even with higher degrees of modification, maintain their cell-adhesive and MMP-degradable peptide sequences, thus avoiding the need for further modification to impart these properties on the hydrogels.

We prepared gelatin derivatives functionalized either by $\mathrm{Tz}$ or $\mathrm{Nb}$ by carbodiimide-based chemistry, ${ }^{44}$ by changing the molar amount of $\mathrm{Tz}$ and $\mathrm{Nb}$ added to functionalize different percentages of the gelatin carboxylic groups. ${ }^{1} \mathrm{H}$ NMR spectroscopy with an internal standard (i.e., TMSP) allowed us to calculate the exact molar amount of $\mathrm{Tz}$ and $\mathrm{Nb}$ bound to gelatin. ${ }^{45}$ Despite a low coupling reaction efficiency (approximately $10 \%$ of $\mathrm{Tz}$ and $\mathrm{Nb}$ bound to gelatin), the percentage DOM was successfully tuned between 5 and $15 \%$ and the obtained DOM was reproducible between independent syntheses $(n>3)$. The gelatin derivatives lost their temperature-dependent gelation properties (Figure S1), which is further confirmation of gelatin modification, as previously demonstrated for decoration of gelatin by $\mathrm{Tz}$ and $\mathrm{Nb}^{44}$ In fact, modification of gelatin might affect its thermoresponsive properties by interfering with the intermolecular forces required to partially reconstitute collagen triple helices during gelatin gelation at $T<T_{\text {sol-gel }}$ depending on the degree of modification, chemistry of the modification, and concentration of gelatin polymer. ${ }^{54,55}$ Despite indirectly confirming gelatin modification, the loss of thermoresponsive properties might represent a limitation for the use of these gelatin derivatives, especially when gelatin temporary transitions are used to prepare constructs with complex shapes by advanced fabrication technologies (e.g., 3D printing on cooled plates). ${ }^{23,56}$

We then investigated the potential versatility of bioorthogonal cross-linked gelatin hydrogels by coupling gelatin derivatives with different DOM (i.e., low $\mathrm{L}$, medium $\mathrm{M}$, and high $\mathrm{H}$ ) at different $\mathrm{Tz} / \mathrm{Nb}$ ratios $R$, after fixing their concentration, and we demonstrated that the rheological 
properties, swelling, and mechanical properties can be tuned by varying the DOM and $R$ ratio. After mixing the gelatin derivatives to form the hydrogels, the rheological properties evolved differently and were especially dependent on the DOM (Figure 3A). The time required for cross-linking (i.e., $\left.t_{50 \% \text { plateau }}\right)$ varied between $10 \mathrm{~min}$ for hydrogels with higher DOM and $70 \mathrm{~min}$, for hydrogels with lower DOM. The varied cross-linking kinetics confirm that, by increasing the DOM of the hydrogels, more $\mathrm{Tz}$ and $\mathrm{Nb}$ molecules are attached to the gelatin derivatives and available to serve as cross-linking sites, thus resulting in faster cross-linking. On the contrary, for lower $\mathrm{DOM}$, the presence of less $\mathrm{Tz}$ and $\mathrm{Nb}$ moieties results in slower cross-linking kinetics. Similar trends in cross-linking kinetics were described for other gelatin cross-linking strategies, where an increase in the amount of cross-linker (e.g., microbial transglutaminase $)^{57}$ or degree of methacryloylation (i.e., GelMA hydrogels) ${ }^{58}$ led to faster cross-linking kinetics. Cross-linking of gelatin hydrogels by $\mathrm{Tz}-\mathrm{Nb}$ chemistry was previously reported to occur faster (i.e., 5-15 min) than our results. ${ }^{44}$ This difference could be attributed to different tetrazine moieties used to prepare the gelatin derivatives. In fact, we employed a methyltetrazine derivative as opposed to the unsubstituted tetrazine derivative used by Koshy et al. (Table S1). ${ }^{44}$ The presence of the methyl group improves tetrazine stability in solution but simultaneously slows its reaction kinetics with norbornene, given its electron donating characteristics. ${ }^{59}$ The slower reaction kinetics of $\mathrm{Tz}-\mathrm{Nb}$ was also confirmed by cross-linking PEG-based hydrogels (i.e., cross-linking time $>2 \mathrm{~h}$ ), compared to other bioorthogonal cross-linking strategies that showed faster cross-linking kinetics (i.e., between seconds and $1 \mathrm{~h}$ ). ${ }^{60}$ Despite the relatively slow cross-linking kinetics, we did observe high cell viability values, which were not affected by the relatively slow cross-linking reaction. Moreover, cells were distributed in $3 \mathrm{D}$ within the hydrogel structure, probably due to the increasing rheological properties, during the ongoing cross-linking, that sustained 3D cell distribution. The faster cross-linking kinetics achieved in other works represents an advantage for some biomedical applications, such as injection of the hydrogels after mixing. ${ }^{44}$ However, the slower cross-linking time achieved here, which might represent a limitation for applications where a quick fixation of the hydrogel structure is required, might be beneficial for other applications, such as when longer manufacturing times are required to process the hydrogel before it is completely cross-linked. ${ }^{61}$

All the hydrogel formulations prepared in this work were structurally compact and could be removed from the PDMS molds and handled after $2 \mathrm{~h}$ of cross-linking. Importantly, their physico-mechanical properties were successfully tuned by changing the $\mathrm{DOM}$ and $\mathrm{Tz} / \mathrm{Nb}$ ratio $R$ used to prepare them. Macroscopically (Figure 2), a higher DOM leads to the formation of more bubbles given by the presence of more $\mathrm{Tz}$ and $\mathrm{Nb}$ molecules that, during their reaction, release $\mathrm{N}_{2}$ as a reaction byproduct. ${ }^{35,36,44}$ Despite the formation of bubbles being an indirect indicator of the successful formation of hydrogels using gelatin derivatives with different DOM values, the presence of bubbles might affect the physico-mechanical properties of the hydrogels. For instance, the presence of bubbles might lead to an underestimation of the mechanical properties of the hydrogels, as their presence might form pores inside hydrogels and lead to decreased mechanical properties compared to bulk hydrogels. ${ }^{23,46,62}$ A more intense pink color was observed by increasing the amount of $\mathrm{Tz}$ gelatin derivative used to prepare the hydrogels (i.e., increasing ratio $R$ ), given by the presence of tetrazine and characterized by a typical pink/ purple color. ${ }^{63}$ At complete swelling, $R 1$ hydrogels are still characterized by a slight pink color that might be attributed to the presence of unreacted $\mathrm{Tz}$. The presence of unreacted $\mathrm{Tz}$ and $\mathrm{Nb}$ can be a consequence of $\mathrm{Tz}$ and $\mathrm{Nb}$ being bound to the gelatin polymer chains that might affect their mobility, thus decreasing the reaction yield. $R 05$ and $R 2$ hydrogels are, on the contrary, characterized by a white and intense pink color, respectively, that confirm the complete reaction of $\mathrm{Tz}$ (and excess $\mathrm{Nb}$ ) and complete reaction of $\mathrm{Nb}$ (and excess $\mathrm{Tz}$ ). After cross-linking, hydrogels were stable in PBS at $37{ }^{\circ} \mathrm{C}$ for 14 days (Figure $3 \mathrm{~B}$ ), proving the successful formation of a cross-linked network of gelatin that would otherwise dissolve in an aqueous environment at $T>T_{\text {sol-gel }}$. Hydrogels swelled differently, as the differently cross-linked networks allowed for different volumes of fluid absorption. Hydrogels prepared with lower DOM and defect of tetrazine (i.e., R05 ratio) were characterized by higher weight variations after immersion in water (i.e., $\Delta w_{\text {DOM L }_{L}}>\Delta w_{\text {DOM M }}$ and $\Delta$ wOM $_{\text {H }} ; \Delta w_{R 05}>$ $\Delta w_{R 1}$ and $\left.\Delta w_{R 2}\right)$. Hydrogels with higher weight variation were also characterized by increased volumetric variation during the swelling (data not shown). Higher degrees of modification showed to decrease gelatin hydrogel swelling which has been observed with other cross-linking strategies, by forming more dense cross-linked structures, including GelMA hydrogels ${ }^{64}$ and acrylamide-cross-linked gelatin hydrogels. ${ }^{24}$ The diverse swelling characteristics of the hydrogels resulted in varied mechanical properties. Hydrogels characterized by a higher weight variation and water absorption were reflected by lower mechanical properties. For instance, GEL_L_R05 hydrogels were characterized by the highest swelling, reflected in the lowest $E$ measured, while GEL_H_R1 and GEL_H_R2 were characterized by the lowest swelling, reflected in the highest measured compressive $E$. The mechanical properties of the hydrogels were tuned (i.e., $E=0.5-5 \mathrm{kPa}$ ) by varying the DOM and $R$ ratio (Figure $3 \mathrm{C}$ ). In general, a higher DOM led to an increase in the mechanical properties (i.e., $E_{\text {DOM } \mathrm{H}_{\mathrm{H}}}>$ $\left.E_{\text {DOM }}>E_{\text {DOM L }}\right)$ and an equimolar $\mathrm{Tz} / \mathrm{Nb}$ ratio increased the mechanical properties (i.e., $E_{R 1}>E_{R 05}$ and $E_{R 2}$ ). Moreover, the mechanical properties of the hydrogels can be correlated to the gel fraction values, as hydrogels retaining higher gelatin solid fraction during swelling were characterized by higher mechanical properties compared to hydrogels with lower percentage gel fraction values (Figure S2B), as similarly demonstrated for GelMA hydrogels. ${ }^{65}$ The higher mechanical properties measured for hydrogels with higher DOM values can be attributed to the formation of an increased number of cross-links, given the higher amount of $\mathrm{Tz}$ and $\mathrm{Nb}$ moieties present on the gelatin derivatives. Interestingly, we did not observe differences in swelling between $R 1$ and $R 2$ hydrogels, despite the formulations being characterized by different mechanical properties. However, the higher mechanical properties detected for $R 1$ hydrogels confirm the different cross-linked hydrogel structure compared to $R 05$ and $R 2$ hydrogels. The higher properties are a consequence of the increased number of cross-links that can be formed when equimolar amounts of $\mathrm{Tz}$ and $\mathrm{Nb}$ (i.e., $R 1$ ) are used to form the hydrogels, compared to $R 05$ and $R 2$ hydrogels where the number of possible cross-links is lower due to residual $\mathrm{Tz}$ and $\mathrm{Nb}$, respectively, that cannot react to form cross-links, thus leading to a weaker hydrogel network. Several strategies have been tested to tailor the properties of gelatin hydrogels, 
including blending with other natural ${ }^{66}$ or synthetic polymers, ${ }^{67}$ preparation of composites, ${ }^{68}$ and variation of the gelatin hydrogel concentration. ${ }^{24,69,70}$ Here, we tuned the mechanical properties of the gelatin hydrogels by changing their cross-linking architecture by using derivatives with different $\mathrm{DOM}$ and changing the $\mathrm{Tz} / \mathrm{Nb}$ ratio. Tuning the properties of gelatin hydrogels by modifying their degree of modification has been reported for GelMA hydrogels (i.e., methacrylation degree), with higher methacryloylation degrees responsible for lower water absorption and higher mechanical properties; ${ }^{64,71-73}$ however, to our knowledge, tuning the properties of gelatin hydrogels cross-linked by $\mathrm{Tz} / \mathrm{Nb}$ chemistry by adjusting the DOM of the gelatin derivatives has not been demonstrated previously. Furthermore, the hydrogels described herein can be cross-linked in a modular fashion without the need for external energy sources (e.g., UV irradiation), thus avoiding potential problems related to depth of penetration of light and UV-related cytotoxic effects. ${ }^{30,31}$

The in vitro response of cells embedded in the hydrogels was modulated depending on their physico-mechanical properties. Importantly, all hydrogels could be degraded after immersion in enzymatic solution, suggesting the presence of MMPsensitive sequences even after gelatin modification and the possible use of the hydrogels as biodegradable scaffolds. We then selected human DPSCs due to their potential versatility in tissue engineering applications. ${ }^{74}$ Dental stem cells share properties with mesenchymal stem cells from bone marrow, and there is a considerable potential for these cells to be used in different stem-cell-based therapies. ${ }^{75}$ In addition, these cells have immunosuppressive-immunomodulatory properties that make them a suitable source for treating immunodisorders. ${ }^{76}$ Moreover, they are easily accessible and an attractive source of autologous or allogeneic stem cells that can be used in the treatment of many clinical conditions in dentistry and medicine. $^{77}$ The tested hydrogel formulations revealed no indirect cytotoxicity (Figure S3), and embedding and culturing viable and metabolically active cells over 14 days of culture was demonstrated (Figure 4). We observed that cells cultured in vitro in hydrogels with lower mechanical properties (i.e., $E<1$ $\mathrm{kPa}$ for GEL_L_R05, GEL_L_R2, and GEL_M_R05) were characterized $\bar{b}$ y higher metabolic activity after $\overline{7}$ and 14 days of culture and by an evident more elongated shape (Figures S5 and 5), compared to cells cultured in hydrogels with higher mechanical properties (i.e., $E>1 \mathrm{kPa}$ for GEL_L_R1, GEL_M_R1, and GEL_M_R2). Higher metabolic activity and increased cell spreading $\bar{h}$ as also been observed for human adipose-derived stem cells encapsulated in gelatin/alginate hydrogels. ${ }^{78}$ Hydrogels with lower stiffness (approximately 3 $\mathrm{kPa}$ ) were also shown to promote cell elongation compared to hydrogels with higher stiffness (approximately $9 \mathrm{kPa}$ ). Similar in vitro cellular response has also been confirmed also by other authors encapsulating human mesenchymal stem cells in gelatin hydrogels ${ }^{79}$ and human umbilical vein endothelial cells in hybrid hyaluronic acid/gelatin hydrogels. ${ }^{80}$ Cells encapsulated in hydrogels with higher matrix stiffness have decreased spreading ability, due to the higher cross-linking and reduced pore size that can affect and/or delay cell spreading. Moreover, despite the possibility that degradation kinetics of the hydrogels might affect cell response, ${ }^{81}$ our data suggest that the differences in cell elongation are more likely to be attributed to the different stiffness values. For instance, the varying stiffness of GEL_M_R05 and GEL_M_R2 hydrogels supported an evident difference in cell morphology, despite the hydrogels being completely degraded within similar timeframes. Moreover, cells embedded in GEL_M_R05 hydrogels were characterized by a more elongated morphology, compared to cells in GEL_L_R1. In this case, the lower stiffness of GEL $M \quad R 05$ compared to GEL L $R 1$ promoted cell elongation, despite GEL_L_R1 being degraded faster than GEL_M_R05.

As the prepared hydrogels displayed promising characteristics as a $3 \mathrm{D}$ biomimetic ECM for in vitro cell culture, we further investigated the possibility of creating more complex hydrogel constructs by chemically coupling gelatin hydrogels. Hydrogels loaded with different cell populations and put in contact one with one another might allow, in the future, for compartmentalized cell coculture, which could be useful for complex tissue regeneration, such as in the case of epithelialmesenchymal cell coculture for organ regeneration by mimicking embryonic morphogenesis, ${ }^{82}$ interface tissue engineering, ${ }^{83}$ and the development of in vitro $3 \mathrm{D}$ coculture models with compartmentalized cocultured cells. ${ }^{84}$ It is however necessary that such hydrogels maintain contact over the in vitro culture period to facilitate cellular processes including migration and paracrine signaling. This could potentially allow for further applications of these hydrogels for compartmentalized cell cocultures. Adhesion between hydrogels was achieved by placing hydrogels prepared with excess $\mathrm{Nb}$ (i.e., R05) and excess $\mathrm{Tz}$ (i.e., R2) in contact without compression. Compared to controls (i.e., coupling of hydrogels with equimolar $R 1 \mathrm{Tz} / \mathrm{Nb}$ ratio and hydrogels crosslinked with glutaraldehyde), the $R 05 \_R 2$ coupled hydrogels could be handled and remained adhered over 14 days in cell culturelike conditions. Moreover, the adhesive tensile strength was approximately doubled when hydrogels with excess $\mathrm{Tz}$ and $\mathrm{Nb}$ were coupled, suggesting the involvement of unreacted excess moieties in keeping the hydrogels adhered. Despite the tensile strength measured for the adhered hydrogels being relatively lower than other gelatin-based adhesive materials described in literature, ${ }^{19,85}$ the adhesive tensile strength between the adhered $R 05 \_R 2$ hydrogels is sufficient to support adhesion between the hydrogels and to allow their manipulation in routine cell culture operation (e.g., tensile strength sufficient to sustain the lifting of the hydrogels weights). Finally, we preliminary tested the possibility of compartmentalized cell culture by loading hDPSCs in R05 hydrogels that were then adhered to acellular $R 2$ hydrogels. After 14 days of culture, the hydrogels were still in contact (Figure S6, left). Cells fully colonized the 3D structure of the R05 hydrogels (Figure S6, top right), as previously evidenced by in vitro tests. Moreover, despite the absence of colonization of the structure of the unseeded $R 2$ hydrogel, we did observe cell migration at the interface between the hydrogels (Figure S6, bottom right), which was confirmed by images of cells on the surface of $R 2$ hydrogels after detaching the two hydrogels (Figure S6, bottom right, inset). These tests show that cellladen hydrogels can be kept in culture while maintaining adhesion to form compartmentalized structures, potentially allowing the future use of the platform here developed for compartmentalized in vitro cocultures.

\section{CONCLUSION}

3D cell-laden gelatin hydrogels were successfully prepared by bioorthogonal cross-linking between tetrazine- and norbornene-gelatin derivatives. The physico-mechanical properties of the hydrogels were varied by tuning the degree of modification 
of the hydrogels and the molar ratio between tetrazine and norbornene, achieving hydrogels with different swelling and rheological and mechanical properties. All the hydrogel formulations were shown to be cytocompatible, as viable cells could be cultured in vitro in the $3 \mathrm{D}$ hydrogels up to 14 days. Higher metabolic activity and cell spreading were observed for hydrogels with lower mechanical properties (approximately $E<1 \mathrm{kPa}$ ). These hydrogels represent promising $3 \mathrm{D}$ culture platforms with tunable properties, do not require external energy sources for cross-linking, can be prepared in mild, cytocompatible conditions, and can modulate cell response depending on the degree of crosslinking. Preliminary evidence of adhesion between hydrogels prepared with $\mathrm{Tz}$ and $\mathrm{Nb}$ excess is given, to potentially further expand the use of such hydrogels for compartmentalized cell cocultures.

\section{ASSOCIATED CONTENT}

\section{SI Supporting Information}

The Supporting Information is available free of charge at https://pubs.acs.org/doi/10.1021/acsbiomaterials.1c00136.

Additional figures showing temperature sweep rheological tests, swelling ratio and gel fraction, indirect in vitro cytotoxicity tests, viability and representative images of cells embedded in the hydrogels, and in vitro compartmentalized cell culture. Table reporting comparison between tetrazine moieties and cross-linking time (PDF)

3D reconstruction of human DPSC cultured in gelatin hydrogels (viable cells in green, dead cells in red) (MP4)

Qualitative adhesion tests between hydrogels (MP4)

Qualitative adhesion tests between hydrogels (MP4)

\section{AUTHOR INFORMATION}

Corresponding Author

Adam D. Celiz - Department of Bioengineering, Imperial College London, W12 0BZ London, U.K.; Email: a.celiz@ imperial.ac.uk

\section{Authors}

Nicola Contessi Negrini - Department of Bioengineering, Imperial College London, W12 OBZ London, U.K.; (1) orcid.org/0000-0003-3719-8869

Ana Angelova Volponi - Centre for Craniofacial and Regenerative Biology, Faculty of Dentistry, King's College London, Guy's Hospital, SE1 9RT London, U.K.

Paul T. Sharpe - Centre for Craniofacial and Regenerative Biology, Faculty of Dentistry, King's College London, Guy's Hospital, SE1 9RT London, U.K.

Complete contact information is available at:

https://pubs.acs.org/10.1021/acsbiomaterials.1c00136

\section{Funding}

This work has been supported by a Confidence in Collaboration in Advanced Therapies Award-Project CiC007 (A.D.C. and P.T.S.) and a UKRI Future Leaders Fellowship MR/S034757/1 (A.D.C.).

\section{Notes}

The authors declare no competing financial interest.

\section{ACKNOWLEDGMENTS}

The authors thank Rousselot Biomedical for providing X-Pure gelatin. The authors thank the Imperial College technical staff for support, in particular S. Patel, S. S. Sangha, and M. A. Hermida Ayala for their assistance with in vitro cell culture, microscopy, and mechanical characterization of the hydrogels.

\section{ABBREVIATIONS}

2D, two-dimensional; 3D, three-dimensional; DPSCs, dental pulp stem cells; ECM, extracellular matrix; EDC, $N$-(3(dimethylamino)propyl)- $N^{\prime}$-ethylcarbodiimide hydrochloride; FBS, fetal bovine serum; GEL, gelatin hydrogel; GELMA, methacryloyl gelatin; HBSS, Hanks' balanced salt solution; MES, 2-(N-morpholino)ethanesulfonic acid; MMP, matrix metalloproteinases; $\mathrm{Nb}$, norbornene; $\mathrm{PBS}$, phosphate buffered saline; PDMS, polydimethylsiloxane; PEG, poly(ethylene glycol); PHEMA, poly(hydroxyethyl methacrylate); PVA, poly(vinyl alcohol); RGD, Arg-Gly-Asp; TCPS, tissue culture polystyrene; Tz, tetrazine; YIGSR, Tyr-Ile-Gly-Ser-Arg

\section{REFERENCES}

(1) Caliari, S. R.; Burdick, J. A. A Practical Guide to Hydrogels for Cell Culture. Nat. Methods 2016, 13 (5), 405-414.

(2) Hedegaard, C. L.; Redondo-Gómez, C.; Tan, B. Y.; Ng, K. W.; Loessner, D.; Mata, A. Peptide-Protein Coassembling Matrices as a Biomimetic 3d Model of Ovarian Cancer. Sci. Adv. 2020, 6 (40), 116.

(3) Vedadghavami, A.; Minooei, F.; Mohammadi, M. H.; Khetani, S.; Rezaei Kolahchi, A.; Mashayekhan, S.; Sanati-Nezhad, A. Manufacturing of Hydrogel Biomaterials with Controlled Mechanical Properties for Tissue Engineering Applications. Acta Biomater. 2017, $62,42-63$.

(4) Livingston, M. K.; Morgan, M. M.; Daly, W. T.; Murphy, W. L.; Johnson, B. P.; Beebe, D. J.; Virumbrales-Muñoz, M. Evaluation of PEG-Based Hydrogel Influence on Estrogen-Receptor-Driven Responses in MCF7 Breast Cancer Cells. ACS Biomater. Sci. Eng. 2019, 5 (11), 6089-6098.

(5) Xin, S.; Gregory, C. A.; Alge, D. L. Interplay between Degradability and Integrin Signaling on Mesenchymal Stem Cell Function within Poly(Ethylene Glycol) Based Microporous Annealed Particle Hydrogels. Acta Biomater. 2020, 101, 227-236.

(6) Luong, T. D.; Zoughaib, M.; Garifullin, R.; Kuznetsova, S.; Guler, M. O.; Abdullin, T. I. In Situ Functionalization of Poly(Hydroxyethyl Methacrylate) Cryogels with Oligopeptides via $\beta$-Cyclodextrin-Adamantane Complexation for Studying Cell-Instructive Peptide Environment. ACS Appl. Bio Mater. 2020, 3, 1116.

(7) Rizwan, M.; Yao, Y.; Gorbet, M. B.; Tse, J. W.; Anderson, D. E. J.; Hinds, M. T.; Yim, E. K. F. One-Pot Covalent Grafting of Gelatin on Poly(Vinyl Alcohol) Hydrogel to Enhance Endothelialization and Hemocompatibility for Synthetic Vascular Graft Applications. ACS Appl. Bio Mater. 2020, 3 (1), 693-703.

(8) Papadimitriou, L.; Manganas, P.; Ranella, A.; Stratakis, E. Biofabrication for Neural Tissue Engineering Applications. Mater. Today Bio 2020, 6, 100043.

(9) Bao, Z.; Xian, C.; Yuan, Q.; Liu, G.; Wu, J. Natural PolymerBased Hydrogels with Enhanced Mechanical Performances: Preparation, Structure, and Property. Adv. Healthcare Mater. 2019, 8 (17), 1900670.

(10) Branco da Cunha, C.; Klumpers, D. D.; Li, W. A.; Koshy, S. T.; Weaver, J. C.; Chaudhuri, O.; Granja, P. L.; Mooney, D. J. Influence of the Stiffness of Three-Dimensional Alginate/Collagen-I Interpenetrating Networks on Fibroblast Biology. Biomaterials 2014, 35 (32), 8927-8936.

(11) Zhu, D.; Wang, H.; Trinh, P.; Heilshorn, S. C.; Yang, F. Elastinlike Protein-Hyaluronic Acid (ELP-HA) Hydrogels with Decoupled 
Mechanical and Biochemical Cues for Cartilage Regeneration. Biomaterials 2017, 127, 132-140.

(12) Spicer, C. D. Hydrogel Scaffolds for Tissue Engineering: The Importance of Polymer Choice. Polym. Chem. 2020, 11 (2), 184-219. (13) Bello, A. B.; Kim, D.; Kim, D.; Park, H.; Lee, S. H. Engineering and Functionalization of Gelatin Biomaterials: From Cell Culture to Medical Applications. Tissue Eng., Part B 2020, 26 (2), 164-180.

(14) Gorgieva, S.; Kokol, V. Collagen- vs. Gelatine-Based Biomaterials and Their Biocompatibility: Review and Perspectives. In Biomaterials Applications for Nanomedicine 2011, 17-52.

(15) Campiglio, C. E.; Contessi Negrini, N.; Farè, S.; Draghi, L. Cross-Linking Strategies for Electrospun Gelatin Scaffolds. Materials 2019, 12 (15), 2476.

(16) van Vlierberghe, S. Crosslinking Strategies for Porous Gelatin Scaffolds. J. Mater. Sci. 2016, 51 (9), 4349.

(17) Cataldo, F.; Ursini, O.; Lilla, E.; Angelini, G. Radiation-Induced Crosslinking of Collagen Gelatin into a Stable Hydrogel. J. Radioanal. Nucl. Chem. 2008, 275 (1), 125-131.

(18) Omata, K.; Matsuno, T.; Asano, K.; Hashimoto, Y.; Tabata, Y.; Satoh, T. Enhanced Bone Regeneration by Gelatin-b-Tricalcium Phosphate Composites Enabling Controlled Release of BFGF. J. Tissue Eng. Regener. Med. 2014, 8, 604-611.

(19) Dinh, T. N.; Hou, S.; Park, S.; Shalek, B. A.; Jeong, K. J. Gelatin Hydrogel Combined with Polydopamine Coating to Enhance Tissue Integration of Medical Implants. ACS Biomater. Sci. Eng. 2018, 4 (10), $3471-3477$.

(20) Wen, C.; Lu, L.; Li, X. Enzymatic and Ionic Crosslinked Gelatin/K-Carrageenan IPN Hydrogels as Potential Biomaterials. J. Appl. Polym. Sci. 2014, 131 (21), 1-8.

(21) Campiglio, C. E.; Ponzini, S.; De Stefano, P.; Ortoleva, G.; Vignati, L.; Draghi, L. Cross-Linking Optimization for Electrospun Gelatin: Challenge of Preserving Fiber Topography. Polymers (Basel, Switz.) 2020, 12 (11), 2472.

(22) Esteves, C.; Santos, G. M. C.; Alves, C.; Palma, S. I. C. J.; Porteira, A. R.; Filho, J.; Costa, H. M. A.; Alves, V. D.; Morais Faustino, B. M.; Ferreira, I.; Gamboa, H.; Roque, A. C. A. Effect of Film Thickness in Gelatin Hybrid Gels for Artificial Olfaction. Mater. Today Bio 2019, 1, 100002.

(23) Contessi Negrini, N.; Celikkin, N.; Tarsini, P.; Farè, S.; Swięszzkowski, W. Three-Dimensional Printing of Chemically Crosslinked Gelatin Hydrogels for Adipose Tissue Engineering. Biofabrication 2020, 12 (2), 025001.

(24) Contessi Negrini, N.; Tarsini, P.; Tanzi, M. C.; Farè, S. Chemically Crosslinked Gelatin Hydrogels as Scaffolding Materials for Adipose Tissue Engineering. J. Appl. Polym. Sci. 2019, 136 (8), 47104 .

(25) Sarem, M.; Arya, N.; Heizmann, M.; Neffe, A. T.; Barbero, A.; Gebauer, T. P.; Martin, I.; Lendlein, A.; Shastri, V. P. Interplay between Stiffness and Degradation of Architectured Gelatin Hydrogels Leads to Differential Modulation of Chondrogenesis in Vitro and in Vivo. Acta Biomater. 2018, 69, 83-94.

(26) Yang, G.; Xiao, Z.; Long, H.; Ma, K.; Zhang, J.; Ren, X.; Zhang, $\mathrm{J}$. Assessment of the Characteristics and Biocompatibility of Gelatin Sponge Scaffolds Prepared by Various Crosslinking Methods. Sci. Rep. 2018, 8 (1), 1-13.

(27) Alcala-Orozco, C. R.; Mutreja, I.; Cui, X.; Kumar, D.; Hooper, G. J.; Lim, K. S.; Woodfield, T. B. F. Design and Characterisation of Multi-Functional Strontium-Gelatin Nanocomposite Bioinks with Improved Print Fidelity and Osteogenic Capacity. Bioprinting 2020, 18 (2019), e00073.

(28) Kerscher, P.; Kaczmarek, J. A.; Head, S. E.; Ellis, M. E.; Seeto, W. J.; Kim, J.; Bhattacharya, S.; Suppiramaniam, V.; Lipke, E. A. Direct Production of Human Cardiac Tissues by Pluripotent Stem Cell Encapsulation in Gelatin Methacryloyl. ACS Biomater. Sci. Eng. 2017, 3 (8), 1499-1509.

(29) Van Hoorick, J.; Tytgat, L.; Dobos, A.; Ottevaere, H.; Van Erps, J.; Thienpont, H.; Ovsianikov, A.; Dubruel, P.; Van Vlierberghe, S. (Photo-)Crosslinkable Gelatin Derivatives for Biofabrication Applications. Acta Biomater. 2019, 97, 46-73.
(30) Lim, K. S.; Klotz, B. J.; Lindberg, G. C. J.; Melchels, F. P. W.; Hooper, G. J.; Malda, J.; Gawlitta, D.; Woodfield, T. B. F. Visible Light Cross-Linking of Gelatin Hydrogels Offers an Enhanced Cell Microenvironment with Improved Light Penetration Depth. Macromol. Biosci. 2019, 19 (6), 1900098.

(31) Nguyen, A. K.; Goering, P. L.; Elespuru, R. K.; Das, S. S.; Narayan, R. J. The Photoinitiator Lithium Phenyl (2,4,6-Trimethylbenzoyl) Phosphinate with Exposure to $405 \mathrm{Nm}$ Light Is Cytotoxic to Mammalian Cells but Not Mutagenic in Bacterial Reverse Mutation Assays. Polymers (Basel, Switz.) 2020, 12 (7), 1489.

(32) Sletten, E. M.; Bertozzi, C. R. From Mechanism to Mouse: A Tale of Two Bioorthogonal Reactions. Acc. Chem. Res. 2011, 44 (9), 666-676.

(33) Arkenberg, M. R.; Nguyen, H. D.; Lin, C. C. Recent Advances in Bio-Orthogonal and Dynamic Crosslinking of Biomimetic Hydrogels. J. Mater. Chem. B 2020, 8 (35), 7835-7855.

(34) Madl, C. M.; Heilshorn, S. C. Bioorthogonal Strategies for Engineering Extracellular Matrices. Adv. Funct. Mater. 2018, 28 (11), 1706046.

(35) Alge, D. L.; Azagarsamy, M. A.; Donohue, D. F.; Anseth, K. S. Synthetically Tractable Click Hydrogels for Three-Dimensional Cell Culture Formed Using Tetrazine-Norbornene Chemistry. Biomacromolecules 2013, 14 (4), 949-953.

(36) Desai, R. M.; Koshy, S. T.; Hilderbrand, S. A.; Mooney, D. J.; Joshi, N. S. Versatile Click Alginate Hydrogels Crosslinked via Tetrazine-Norbornene Chemistry. Biomaterials 2015, 50 (1), 30-37.

(37) Delplace, V.; Nickerson, P. E. B.; Ortin-Martinez, A.; Baker, A. E. G.; Wallace, V. A.; Shoichet, M. S. Nonswelling, Ultralow Content Inverse Electron-Demand Diels-Alder Hyaluronan Hydrogels with Tunable Gelation Time: Synthesis and In Vitro Evaluation. Adv. Funct. Mater. 2020, 30 (14), 1903978.

(38) Delplace, V.; Pickering, A. J.; Hettiaratchi, M. H.; Zhao, S.; Kivijärvi, T.; Shoichet, M. S. Inverse Electron-Demand Diels-Alder Methylcellulose Hydrogels Enable the Co-Delivery of Chondroitinase $\mathrm{ABC}$ and Neural Progenitor Cells. Biomacromolecules 2020, 21 (6), 2421-2431.

(39) Carthew, J.; Frith, J. E.; Forsythe, J. S.; Truong, V. X. Polyethylene Glycol-Gelatin Hydrogels with Tuneable Stiffness Prepared by Horseradish Peroxidase-Activated Tetrazine-Norbornene Ligation. J. Mater. Chem. B 2018, 6 (9), 1394-1401.

(40) Jivan, F.; Yegappan, R.; Pearce, H.; Carrow, J. K.; McShane, M.; Gaharwar, A. K.; Alge, D. L. Sequential Thiol-Ene and Tetrazine Click Reactions for the Polymerization and Functionalization of Hydrogel Microparticles. Biomacromolecules 2016, 17 (11), 3516-3523.

(41) Zhang, Z.; He, C.; Chen, X. Injectable Click Polypeptide Hydrogels via Tetrazine-Norbornene Chemistry for Localized Cisplatin Release. Polymers (Basel, Switz.) 2020, 12 (4), 884.

(42) Truong, V. X.; Ablett, M. P.; Richardson, S. M.; Hoyland, J. A.; Dove, A. P. Simultaneous Orthogonal Dual-Click Approach to Tough, in-Situ -Forming Hydrogels for Cell Encapsulation. J. Am. Chem. Soc. 2015, 137 (4), 1618-1622.

(43) Truong, V. X.; Ablett, M. P.; Gilbert, H. T. J.; Bowen, J.; Richardson, S. M.; Hoyland, J. A.; Dove, A. P. In Situ-Forming Robust Chitosan-Poly(Ethylene Glycol) Hydrogels Prepared by Copper-Free Azide-Alkyne Click Reaction for Tissue Engineering. Biomater. Sci. 2014, 2 (2), 167-175.

(44) Koshy, S. T.; Desai, R. M.; Joly, P.; Li, J.; Bagrodia, R. K.; Lewin, S. A.; Joshi, N. S.; Mooney, D. J. Click-Crosslinked Injectable Gelatin Hydrogels. Adv. Healthcare Mater. 2016, 5 (5), 541-547.

(45) Claaßen, C.; Claaßen, M. H.; Truffault, V.; Sewald, L.; Tovar, G. E. M.; Borchers, K.; Southan, A. Quantification of Substitution of Gelatin Methacryloyl: Best Practice and Current Pitfalls. Biomacromolecules 2018, 19 (1), 42-52.

(46) Contessi Negrini, N.; Bonnetier, M.; Giatsidis, G.; Orgill, D. P. D. P.; Farè, S.; Marelli, B. Tissue-Mimicking Gelatin Scaffolds by Alginate Sacrificial Templates for Adipose Tissue Engineering. Acta Biomater. 2019, 87, 61-75.

(47) Marelli, B.; Le Nihouannen, D.; Hacking, S. A.; Tran, S.; Li, J.; Murshed, M.; Doillon, C. J.; Ghezzi, C. E.; Zhang, Y. L.; Nazhat, S. 
N.; Barralet, J. E. Newly Identified Interfibrillar Collagen Crosslinking Suppresses Cell Proliferation and Remodelling. Biomaterials 2015, 54, 126-135.

(48) Michelini, L.; Probo, L.; Farè, S.; Contessi Negrini, N. Characterization of Gelatin Hydrogels Derived from Different Animal Sources. Mater. Lett. 2020, 272, 127865.

(49) Yuk, H.; Varela, C. E.; Nabzdyk, C. S.; Mao, X.; Padera, R. F. Dry Double-Sided Tape for Adhesion of Wet Tissues and Devices. Nature 2019, 575, 169.

(50) Li, C.; Liu, I. K. K.; Tsao, C. Y.; Chan, V. Neuronal Differentiation of Human Placenta-Derived Multi-Potent Stem Cells Enhanced by Cell Body Oscillation on Gelatin Hydrogel. J. Bioact. Compat. Polym. 2014, 29 (6), 529-544.

(51) Sirova, M.; Vlierberghe, S.; Van Matyasova, V.; Rossmann, P.; Schacht, E.; Dubruel, P.; Rihova, B. Immunocompatibility Evaluation of Hydrogel-Coated Polyimide Implants for Applications in Regenerative Medicine. J. Biomed. Mater. Res., Part A 2014, 102 (6), 1982-1990.

(52) Pahoff, S.; Meinert, C.; Bas, O.; Nguyen, L.; Klein, T. J.; Hutmacher, D. W. Effect of Gelatin Source and Photoinitiator Type on Chondrocyte Redifferentiation in Gelatin Methacryloyl-Based Tissue-Engineered Cartilage Constructs. J. Mater. Chem. B 2019, 7 (10), 1761-1772

(53) Groen, W. M. G. A. C.; Utomo, L.; Castilho, M.; Gawlitta, D.; Malda, J.; van Weeren, P. R.; Levato, R.; Korthagen, N. M. Impact of Endotoxins in Gelatine Hydrogels on Chondrogenic Differentiation and Inflammatory Cytokine Secretion in Vitro. Int. J. Mol. Sci. 2020, 21 (22), 8571.

(54) Hoch, E.; Hirth, T.; Tovar, G. E. M.; Borchers, K. Chemical Tailoring of Gelatin to Adjust Its Chemical and Physical Properties for Functional Bioprinting. J. Mater. Chem. B 2013, 1 (41), 56755685.

(55) Sewald, L.; Claaßen, C.; Götz, T.; Claaßen, M. H.; Truffault, V.; Tovar, G. E. M.; Southan, A.; Borchers, K. Beyond the Modification Degree: Impact of Raw Material on Physicochemical Properties of Gelatin Type A and Type B Methacryloyls. Macromol. Biosci. 2018, 18 (12), 1800168.

(56) Koch, F.; Tröndle, K.; Finkenzeller, G.; Zengerle, R.; Zimmermann, S.; Koltay, P. Generic Method of Printing Window Adjustment for Extrusion-Based 3D-Bioprinting to Maintain High Viability of Mesenchymal Stem Cells in an Alginate-Gelatin Hydrogel. Bioprinting 2020, 20 (April), e00094.

(57) Zhou, M.; Lee, B. H.; Tan, Y. J.; Tan, L. P. Microbial Transglutaminase Induced Controlled Crosslinking of Gelatin Methacryloyl to Tailor Rheological Properties for 3D Printing. Biofabrication 2019, 11 (2), 025011.

(58) Pepelanova, I.; Kruppa, K.; Scheper, T.; Lavrentieva, A. GelatinMethacryloyl (GelMA) Hydrogels with Defined Degree of Functionalization as a Versatile Toolkit for 3D Cell Culture and Extrusion Bioprinting. Bioengineering 2018, 5 (3), 55.

(59) Karver, M. R.; Weissleder, R.; Hilderbrand, S. A. Synthesis and Evaluation of a Series of 1,2,4,5-Tetrazines for Bioorthogonal Conjugation. Bioconjugate Chem. 2011, 22 (11), 2263-2270.

(60) Zhan, H.; De Jong, H.; Löwik, D. W. P. M. Comparison of Bioorthogonally Cross-Linked Hydrogels for in Situ Cell Encapsulation. ACS Appl. Bio Mater. 2019, 2 (7), 2862-2871.

(61) Madl, C. M.; Katz, L. M.; Heilshorn, S. C. Bio-Orthogonally Crosslinked, Engineered Protein Hydrogels with Tunable Mechanics and Biochemistry for Cell Encapsulation. Adv. Funct. Mater. 2016, 26 (21), 3612-3620.

(62) Hwang, C. M.; Sant, S.; Masaeli, M.; Kachouie, N. N.; Zamanian, B.; Lee, S. H.; Khademhosseini, A. Fabrication of ThreeDimensional Porous Cell-Laden Hydrogel for Tissue Engineering. Biofabrication 2010, 2 (3), 035003.

(63) Hansell, C. F.; Espeel, P.; Stamenovic, M. M.; Barker, I. A.; Dove, A. P.; Du Prez, F. E.; O’Reilly, R. K. Additive-Free Clicking for Polymer Functionalization and Coupling by Tetrazine-Norbornene Chemistry. J. Am. Chem. Soc. 2011, 133 (35), 13828-13831.
(64) Zhu, M.; Wang, Y.; Ferracci, G.; Zheng, J.; Cho, N. J.; Lee, B. $\mathrm{H}$. Gelatin Methacryloyl and Its Hydrogels with an Exceptional Degree of Controllability and Batch-to-Batch Consistency. Sci. Rep. 2019, 9 (1), 1-13.

(65) Van Nieuwenhove, I.; Salamon, A.; Peters, K.; Graulus, G. J.; Martins, J. C.; Frankel, D.; Kersemans, K.; De Vos, F.; Van Vlierberghe, S.; Dubruel, P. Gelatin- and Starch-Based Hydrogels. Part A: Hydrogel Development, Characterization and Coating. Carbohydr. Polym. 2016, 152, 129-139.

(66) Rinoldi, C.; Fallahi, A.; Yazdi, I. K.; Campos Paras, J.; KijeńskaGawrońska, E.; Trujillo-De Santiago, G.; Tuoheti, A.; Demarchi, D.; Annabi, N.; Khademhosseini, A.; Swieszkowski, W.; Tamayol, A. Mechanical and Biochemical Stimulation of 3D Multilayered Scaffolds for Tendon Tissue Engineering. ACS Biomater. Sci. Eng. 2019, 5 (6), 2953-2964.

(67) Liang, J.; Guo, Z.; Timmerman, A.; Grijpma, D.; Poot, A. Enhanced Mechanical and Cell Adhesive Properties of PhotoCrosslinked PEG Hydrogels by Incorporation of Gelatin in the Networks. Biomed. Mater. 2019, 14 (2), 024102.

(68) Nosrati, H.; Sarraf Mamoory, R.; Svend Le, D. Q.; Bünger, C. E. Fabrication of Gelatin/Hydroxyapatite/3D-Graphene Scaffolds by a Hydrogel 3D-Printing Method. Mater. Chem. Phys. 2020, 239, 122305.

(69) Celikkin, N.; Mastrogiacomo, S.; Jaroszewicz, J.; Walboomers, X. F.; Swieszkowski, W. Gelatin Methacrylate Scaffold for Bone Tissue Engineering: The Influence of Polymer Concentration. J. Biomed. Mater. Res., Part A 2018, 106 (1), 201-209.

(70) Van Hoorick, J.; Gruber, P.; Markovic, M.; Tromayer, M.; Van Erps, J.; Thienpont, H.; Liska, R.; Ovsianikov, A.; Dubruel, P.; Van Vlierberghe, S. Cross-Linkable Gelatins with Superior Mechanical Properties Through Carboxylic Acid Modification: Increasing the Two-Photon Polymerization Potential. Biomacromolecules 2017, 18 (10), 3260-3272.

(71) Li, X.; Chen, S.; Li, J.; Wang, X.; Zhang, J.; Kawazoe, N.; Chen, G. D Culture of Chondrocytes in Gelatin Hydrogels with Different Stiffness. Polymers (Basel, Switz.) 2016, 8 (8), 269.

(72) Nichol, J. W.; Koshy, S. T.; Bae, H.; Hwang, C. M.; Yamanlar, S.; Khademhosseini, A. Cell-Laden Microengineered Gelatin Methacrylate Hydrogels. Biomaterials 2010, 31 (21), 5536-5544.

(73) Irmak, G.; Demirtaş, T. T.; Gumusderelioglu, M. Highly Methacrylated Gelatin Bioink for Bone Tissue Engineering. ACS Biomater. Sci. Eng. 2019, 5 (2), 831-845.

(74) Nuti, N.; Corallo, C.; Chan, B. M. F.; Ferrari, M.; GeramiNaini, B. Multipotent Differentiation of Human Dental Pulp Stem Cells: A Literature Review. Stem Cell Rev. Reports 2016, 12 (5), 511523.

(75) Volponi, A. A.; Pang, Y.; Sharpe, P. T. Stem Cell-Based Biological Tooth Repair and Regeneration. Trends Cell Biol. 2010, 20 (12), 715-722.

(76) Yamaza, T.; Kentaro, A.; Chen, C.; Liu, Y.; Shi, Y.; Gronthos, S.; Wang, S.; Shi, S. Immunomodulatory Properties of Stem Cells from Human Exfoliated Deciduous Teeth. Stem Cell Res. Ther. 2010, 1 (1), 5 .

(77) Angelova Volponi, A.; Zaugg, L. K.; Neves, V.; Liu, Y.; Sharpe, P. T. Tooth Repair and Regeneration. Curr. Oral Heal. Reports 2018, 5 (4), 295-303.

(78) Sarker, B.; Zehnder, T.; Rath, S. N.; Horch, R. E.; Kneser, U.; Detsch, R.; Boccaccini, A. R. Oxidized Alginate-Gelatin Hydrogel: A Favorable Matrix for Growth and Osteogenic Differentiation of Adipose-Derived Stem Cells in 3D. ACS Biomater. Sci. Eng. 2017, 3 (8), 1730-1737.

(79) Mũnoz, Z.; Shih, H.; Lin, C. C. Gelatin Hydrogels Formed by Orthogonal Thiol-Norbornene Photochemistry for Cell Encapsulation. Biomater. Sci. 2014, 2 (8), 1063-1072.

(80) Camci-Unal, G.; Cuttica, D.; Annabi, N.; Demarchi, D.; Khademhosseini, A. Synthesis and Characterization of Hybrid Hyaluronic Acid-Gelatin Hydrogels. Biomacromolecules 2013, 14 (4), 1085-1092. 
(81) Tsou, Y. H.; Khoneisser, J.; Huang, P. C.; Xu, X. Hydrogel as a Bioactive Material to Regulate Stem Cell Fate. Bioact. Mater. 2016, 1 (1), 39-55.

(82) Contessi Negrini, N.; Angelova Volponi, A.; Higgins, C.A.; Sharpe, P.T.; Celiz, A.D. Scaffold-Based Developmental Tissue Engineering Strategies for Ectodermal Organ Regeneration. Mater. Today Bio 2021, 10, 100107.

(83) Nooeaid, P.; Roether, J. A.; Weber, E.; Schubert, D. W.; Boccaccini, A. R. Technologies for Multilayered Scaffolds Suitable for Interface Tissue Engineering. Adv. Eng. Mater. 2014, 16 (3), 319327.

(84) Nii, T.; Makino, K.; Tabata, Y. Three-Dimensional Culture System of Cancer Cells Combined with Biomaterials for Drug Screening. Cancers 2020, 12 (10), 2754.

(85) Zhao, Q.; Mu, S.; Long, Y.; Zhou, J.; Chen, W.; Astruc, D.; Gaidau, C.; Gu, H. Tannin-Tethered Gelatin Hydrogels with Considerable Self-Healing and Adhesive Performances. Macromol. Mater. Eng. 2019, 304 (4), 1800664. 